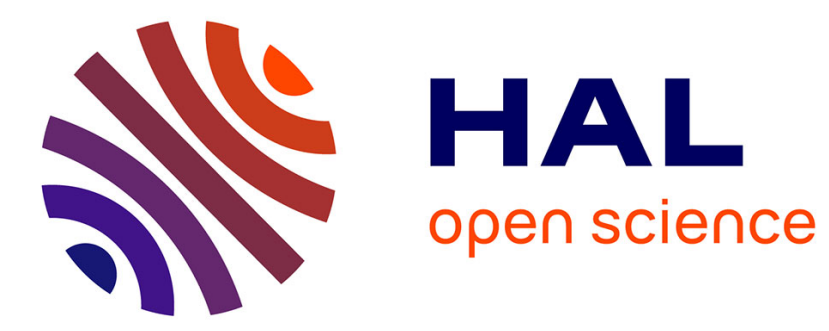

\title{
Light-assisted dynamic titration: From theory to an experimental protocol
}

Agnès Pellissier-Tanon, Raja Chouket, Thomas Le Saux, Ludovic Jullien, Annie Lemarchand

\section{- To cite this version:}

Agnès Pellissier-Tanon, Raja Chouket, Thomas Le Saux, Ludovic Jullien, Annie Lemarchand. Lightassisted dynamic titration: From theory to an experimental protocol. Physical Chemistry Chemical Physics, 2018, 20 (37), pp.23998 - 24010. 10.1039/C8CP03953D . hal-01913670

\section{HAL Id: hal-01913670 \\ https://hal.sorbonne-universite.fr/hal-01913670}

Submitted on 6 Nov 2018

HAL is a multi-disciplinary open access archive for the deposit and dissemination of scientific research documents, whether they are published or not. The documents may come from teaching and research institutions in France or abroad, or from public or private research centers.
L'archive ouverte pluridisciplinaire HAL, est destinée au dépôt et à la diffusion de documents scientifiques de niveau recherche, publiés ou non, émanant des établissements d'enseignement et de recherche français ou étrangers, des laboratoires publics ou privés. 


\title{
Light-assisted dynamic titration: From theory to an experimental protocol
}

\author{
Agnès Pellissier-Tanon, ${ }^{1}$ Raja Chouket, ${ }^{1}$ Thomas Le Saux,${ }^{1}$ \\ Ludovic Jullien, ${ }^{1, a)}$ and Annie Lemarchand ${ }^{2, b)}$ \\ ${ }^{1}$ PASTEUR, Département de Chimie, École normale supérieure, \\ PSL University, Sorbonne Université, CNRS, 75005 Paris, France. \\ ${ }^{2}$ Sorbonne Université, Centre National de la Recherche Scientifique (CNRS) \\ Laboratoire de Physique Théorique de la Matière Condensée (LPTMC), \\ 4 place Jussieu, case courrier 121, 75252 Paris Cedex 05, France.
}

September 3, 2018

\begin{abstract}
In order to design a dynamic titration method, we propose a theoretical model harnessing the kinetic properties of the complexation of the titrated species with a titrating photoswitchable reagent. Forced oscillations of illumination are imposed and concentration oscillations of the targeted species are deduced from the equations of chemical kinetics. We determine analytical expressions of the resonance conditions on the control parameters, angular frequency, mean light intensity, and total concentration of the photoswitchable reagent, which optimize the out-of-phase amplitude of concentration oscillations. A user-friendly protocol of dynamic titration is proposed.
\end{abstract}

a) Author to whom correspondence should be addressed. Electronic mail: ludovic.jullien@ens.fr

b) Author to whom correspondence should be addressed. Electronic mail: anle@lptmc.jussieu.fr 


\section{Introduction}

In analysis or imaging, addressing a targeted component in a mixture without any separation requires selectively reading-out its signature. When a mixture contains many components, exploiting a spectroscopic signature may be too restrictive for discrimination. Indeed, the signals associated with atoms or functional groups exhibit non-vanishing half-widths and are spread over a finite frequency range, which limits the number of discernable components. As an example relevant for fluorescence microscopy, ${ }^{1}$ the absorption and fluorescence emission features of the labels currently used in biology suffer from spectral crowding. Only a few labels can be discriminated $^{2}$ instead of the several tens which would be desirable for advanced bioimaging, ${ }^{3,4}$ multicolored cell labeling, ,,6,7 and highly multiplexed diagnostic assays. ${ }^{8,9}$ Alternative strategies have to be implemented in order to overcome the preceding limitation.

Titration is such a strategy, which has been extensively used in chemistry and biology. In titrations, discrimination of a targeted component relies on its reaction with a selective reagent, which is read-out by an observable (e.g. absorbance, fluorescence emission,...) reporting on the extent of the titrating reaction. Most titration protocols exploit chemical equilibrium with respect to the titrating reaction. In those protocols, the observable is constant and its value is governed by the thermodynamic constant of the titrating reaction, which has to be singularized in order to achieve successful contrast of the targeted component against interfer-

ing species. Dynamic titrations add a new dimension for discrimination. ${ }^{10,11}$ As relaxation methods used in chemical kinetics, ${ }^{12,13,14,15,16}$ they exploit the mixture response to a sudden or periodic change of a parameter, which controls the rate of the titrating reaction. In dynamic titrations, the observable is not anymore constant but it is replaced by a temporal series of observable values, which depends on the impact of the perturbation on the titrating reaction. In particular, both the extent 
and the relaxation time of the titrating reaction govern the response of any mixture component to the perturbation. Consequently, they add a kinetic dimension to the titration protocols relevant at chemical equilibrium and correspondingly make dynamic titrations more selective.

Several control parameters can perturb the rate of a titration reaction. ${ }^{12,}{ }^{14} \mathrm{Pe}-$ riodic forcing of reaction kinetics via, e.g. modulation of pressure, temperature, or illumination, is a useful tool to improve the reaction performance, the reaction rate, or to control the selection of a given behavior in complex nonlinear reactions. ${ }^{17,18,19,20,21}$ Optimizing the quantity of interest consists in determining resonance conditions relating the parameters associated with external forcing and intrinsic chemical dynamics. Analogous optimization could be obtained using noise instead of a periodic forcing leading to stochastic resonance. ${ }^{22}$ Protocols relying on temperature modulations and signal demodulation for selective analysis ${ }^{23,24,25}$ or mechanism unraveling 26, 27, 28, 29, 30,31,32,33 have been proposed.

In this manuscript, we consider perturbing the rate of the titration reaction by changing the concentration of its reactants and products. Such a change would most often involve matter transport. However, this approach may bring limitations originating from poor temporal resolution, detrimental convective flows, or lack of access in the case of compartmentalized systems. We are interested in using light as a fast non-invasive trigger to change the concentration of photoactive species, which is compatible with biological applications. ${ }^{34}$ Hence, we consider that the selective reagent used in the titration is reversibly photoswitchable and exchanges between two states respectively reactive and inert towards the mixture components. Our goal is to introduce a highly selective dynamic titration protocol discriminating the mixture components by the rate constants of their reaction with the reactive state of the selective reagent. Interestingly, the same protocol will be as well relevant to add another dimension to discriminate recent biomolecular tags, which non-covalently in- 
teract with reversibly photoswitchable reagents to provide fluorescent labels. ${ }^{35,36,37}$

The paper is organized as follows. In Section 2, we introduce a model of dynamic titration involving a photoswitchable reagent and forced oscillations of illumination. In Section 3, we analytically compute the concentration response of the reactants and products engaged in the light-assisted titrating reaction when they are submitted to modulated illumination of small amplitude. The observable is the out-of-phase amplitude of concentration oscillations of the complex formed by the targeted species and the photoswitchable titrating reagent. The observable displays an optimum in the space of the rate constants. These resonant rate constants are associated with specific values of the control parameters, i.e. the angular frequency of illumination modulation, the mean light intensity and the concentration of the photoswitchable reagent. Reciprocally, setting the control parameters to resonant values leads to singularizing the species with kinetic properties of interest. In Section 4, we extract analytical expressions of the control parameters associated with an extremum of the observable. The analytical predictions are numerically checked in Section 5. Section 6 is devoted to the design of the experimental protocol for light-assisted dynamic titration. Section 7 contains the conclusion.

\section{The model}

We consider the following mechanism accounting for the photochemical isomerization of a reversibly photoswitchable reagent $(\mathbf{1 F}, \mathbf{2 F})$ and its complexation with a titrated species $\mathbf{R}$ :

$$
\begin{aligned}
& \mathbf{1 F} \stackrel{k_{12}^{h \nu}}{\rightleftharpoons} \quad \mathbf{2 F} \\
& k_{21}^{h \nu}+k_{21}^{\Delta} \\
& \mathbf{R}+\mathbf{1 F} \stackrel{k_{+1}^{\Delta}}{\rightleftharpoons} \quad \mathbf{B} \\
& k_{-1}^{\Delta}
\end{aligned}
$$


In reaction (1), the thermodynamically most stable state $\mathbf{1 F}$ is photochemically converted into the thermodynamically less stable state $\mathbf{2 F}$ at rate constant $k_{12}^{h \nu}$ from which it can relax back to the initial state $\mathbf{1 F}$ either by a photochemicallyor a thermally-driven process at rate constant $k_{21}^{h \nu}+k_{21}^{\Delta}$, where $k_{21}^{h \nu}$ and $k_{21}^{\Delta}$ are the photochemical and the thermal contributions of the rate constants, respectively. In reaction (2), the state $\mathbf{1 F}$ interacts with the titrated species $\mathbf{R}$ to yield the bound, fluorescent state 1B. The thermally-driven on- and off-rate constants, $k_{+1}^{\Delta}$ and $k_{-1}^{\Delta}$, are used as discriminative parameters in a dynamic titration protocol.

In this work, the photochemical reaction (1) is used to drive the complexation reaction (2) and reveal its kinetics. Numerous molecular backbones exhibit reversible photoswitching when they are exposed to light. ${ }^{38,39,40,41}$ The forward and backward photochemical isomerizations of reaction (1) can be light-driven at a same wavelength. Alternatively two distinct wavelengths can be used to respectively govern the forward and backward photochemical processes in many reversibly photoswitchable chromophores (e.g. reversibly fluorescent photoswitchable proteins - RSFPs, ${ }^{42,} 43$ azobenzenes, ${ }^{44,45,46}$ cyanines, ${ }^{47,} 48$ diarylethenes, ${ }^{49,50,51}$ and spirobenzopyrans $\left.{ }^{52}\right)$. In the following, we distinguish two regimes of illumination. In the first regime, subsequently referred to as the one-color system, we consider that the forward and backward photochemical isomerizations are driven at one wavelength only. In this regime, the thermally-driven backward isomerization associated with the rate constant $k_{21}^{\Delta}$ is essential to control the modulation of the extent of reaction (1). In the second regime, subsequently denominated as the two-color system, we assume that the forward and backward photochemical isomerizations are driven at two distinct wavelengths. The thermally-driven backward isomerization is not necessary to control the modulation of the extent of reaction (1) and we restrict the examination of the two-color system to the case $k_{21}^{\Delta} \ll k_{21}^{h \nu}$, i.e. to high intensities of illumination. 


\section{Concentration responses to small oscillations of illumination}

We investigate the response of the system to forced oscillations of the rate constants $k_{12}^{h \nu}$ and $k_{21}^{h \nu}$ around their steady values $k_{12}^{0}$ and $k_{21}^{0}$. Denoting $1 B, R, 1 F$, and $2 F$ the concentrations in $\mathbf{1 B}, \mathbf{R}, \mathbf{1 F}$, and $\mathbf{2} \mathbf{F}$ respectively, we express the laws of conservation of matter

$$
\begin{aligned}
& R_{t o t}=1 B+R \\
& F_{t o t}=1 F+2 F+1 B
\end{aligned}
$$

where $R_{t o t}$ and $F_{\text {tot }}$ denote the total concentrations of the titrated species and the photoswitchable reagent, respectively. The temporal evolution of the concentrations is governed by

$$
\begin{aligned}
\frac{d 1 B}{d t}= & k_{+1}^{\Delta} 1 B^{2}+k_{+1}^{\Delta} 1 B 2 F-\left[k_{+1}^{\Delta}\left(R_{t o t}+F_{t o t}\right)+k_{-1}^{\Delta}\right] 1 B \\
& -k_{+1}^{\Delta} R_{t o t} 2 F+k_{+1}^{\Delta} R_{t o t} F_{t o t} \\
\frac{d 2 F}{d t}= & -k_{12}^{h \nu} 1 B-\left(k_{12}^{h \nu}+k_{21}^{h \nu}+k_{21}^{\Delta}\right) 2 F+k_{12}^{h \nu} F_{t o t}
\end{aligned}
$$

In the one-color system, photoswitching is driven at one wavelength. With a one-photon excitation, the photochemical rate constants can be written

$$
\begin{aligned}
& k_{12}^{h \nu}(t)=\sigma_{12} I(t) \\
& k_{21}^{h \nu}(t)=\sigma_{21} I(t)
\end{aligned}
$$

where $\sigma_{12}$ and $\sigma_{21}$ are the photoswitching cross-sections and $I(t)$ is the light intensity. In the two-color system, photoswitching is driven at two wavelengths with associated light intensities $I_{1}(t)$ and $I_{2}(t)$. The rate constants obey

$$
\begin{aligned}
& k_{12}^{h \nu}(t)=\sigma_{12,1} I_{1}(t)+\sigma_{12,2} I_{2}(t) \\
& k_{21}^{h \nu}(t)=\sigma_{21,1} I_{1}(t)+\sigma_{21,2} I_{2}(t)
\end{aligned}
$$


where $\sigma_{12,1}, \sigma_{12,2}, \sigma_{21,1}$, and $\sigma_{21,2}$ are the photoswitching cross-sections. The thermal rate constant $k_{21}^{\Delta}$ is neglected in front of the photochemical rate constants, implying that the light intensities are large enough.

\subsection{Steady-state concentrations}

In this subsection, we consider steady illumination, $I(t)=I^{0}$ for the one-color system and $I_{1}(t)=I_{1}^{0}$ and $I_{2}(t)=I_{2}^{0}$ for the two-color system. We look for the steady solutions of Eqs. $(5,6)$ for stationary values of the photochemical rate constants

$$
\begin{aligned}
& k_{12}^{0}=\sigma_{12} I^{0} \\
& k_{21}^{0}=\sigma_{21} I^{0}
\end{aligned}
$$

for the one-color system and

$$
\begin{aligned}
& k_{12}^{0}=\sigma_{12,1} I_{1}^{0}+\sigma_{12,2} I_{2}^{0} \\
& k_{21}^{0}=\sigma_{21,1} I_{1}^{0}+\sigma_{21,2} I_{2}^{0}
\end{aligned}
$$

for the two-color system. We introduce dimensionless steady concentrations

$$
\begin{aligned}
& \Psi_{1 B}^{0}=\frac{k_{+1}^{\Delta}}{k_{-1}^{\Delta}} 1 B^{0} \\
& \Psi_{2 F}^{0}=\frac{k_{+1}^{\Delta}}{k_{-1}^{\Delta}} 2 F^{0}
\end{aligned}
$$

which obey for the one-color system

$$
\begin{aligned}
\Psi_{1 B}^{0}{ }^{2}+\Psi_{1 B}^{0} \Psi_{2 F}^{0}- & \left(\zeta_{R}+\zeta_{F}+1\right) \Psi_{1 B}^{0}-\zeta_{R} \Psi_{2 F}^{0}+\zeta_{R} \zeta_{F}=0 \\
-\eta \Psi_{1 B}^{0}-[\eta(\gamma+1)+1] \Psi_{2 F}^{0}+\eta \zeta_{F} & =0
\end{aligned}
$$

and depend on the four dimensionless parameters $\zeta_{R}, \zeta_{F}, \eta$, and $\gamma$ given in Table 1. The dimensionless steady concentrations are

$$
\begin{aligned}
& \Psi_{1 B}^{0}=\zeta_{F}-\frac{\eta(1+\gamma)+1}{\eta} \Psi_{2 F}^{0} \\
& \Psi_{2 F}^{0}=\frac{-f+\sqrt{f^{2}-4 e g}}{2 e}
\end{aligned}
$$




\begin{tabular}{|c|c|c|}
\hline & One-color system & Two-color system \\
\hline$\zeta_{R}$ & $\frac{k_{+1}^{\Delta}}{k^{\Delta}} R_{t o t}$ & $\frac{k_{+1}^{\Delta}}{k_{-1}^{\Delta}} R_{t o t}$ \\
$\zeta_{F}$ & $\frac{k_{+1}}{k_{-1}} F_{t o t}$ & $\frac{k_{+1}}{k_{\Delta}} F_{t o t}$ \\
$\eta$ & $\frac{\sigma_{12} I^{0}}{k_{21}}$ & $\frac{\sigma_{21,1} I_{1}^{0}}{\sigma_{21,2} I_{2}^{0}}$ \\
$\gamma$ & $\frac{\sigma_{21}}{\sigma_{12}}$ & $\frac{\sigma_{12,1}}{\sigma_{21,1}}$ \\
$\gamma_{1}$ & & $\frac{\sigma_{12,2}}{\sigma_{21,2}}$ \\
$\gamma_{2}$ & & \multicolumn{2}{|c}{} \\
\hline
\end{tabular}

Table 1: Expression of the dimensionless parameters $\zeta_{R}, \zeta_{F}, \eta, \gamma, \gamma_{1}$, and $\gamma_{2}$ for the one- and two-color systems.

\begin{tabular}{|c|c|c|}
\hline & One-color system & Two-color system \\
\hline$e$ & $(\eta(1+\gamma)+1)(\eta \gamma+1)$ & $(\eta+1)\left(\eta\left(\gamma_{1}+1\right)+\gamma_{2}+1\right)$ \\
$f$ & $\eta\left(\left(\zeta_{R}-\zeta_{F}\right)(\eta \gamma+1)+\eta(1+\gamma)+1\right)$ & $\left(\eta \gamma_{1}+\gamma_{2}\right)\left(\left(\zeta_{R}-\zeta_{F}\right)(\eta+1)+\eta\left(\gamma_{1}+1\right)+\gamma_{2}+1\right)$ \\
$g$ & $-\eta^{2} \zeta_{F}$ & $-\left(\eta \gamma_{1}+\gamma_{2}\right)^{2} \zeta_{F}$ \\
\hline
\end{tabular}

Table 2: Expression of the parameters $e, f$, and $g$ for the one- and two-color systems where $\zeta_{F}, \eta, \gamma, \gamma_{1}$, and $\gamma_{2}$ are given in table 1 .

with $e, f$, and $g$ given in Table 2 .

For the two-color system, Eqs. $(17,20)$ remain unchanged and Eqs.(18,19) are substituted by

$$
\begin{aligned}
0 & =-\left(\eta \gamma_{1}+\gamma_{2}\right) \Psi_{1 B}^{0}-\left[\eta\left(\gamma_{1}+1\right)+\gamma_{2}+1\right] \Psi_{2 F}^{0}+\left(\eta \gamma_{1}+\gamma_{2}\right) \zeta_{F} \\
\Psi_{1 B}^{0} & =\zeta_{F}-\left(1+\frac{\eta+1}{\eta \gamma_{1}+\gamma_{2}}\right) \Psi_{2 F}^{0}
\end{aligned}
$$

where the expressions $\eta, \gamma_{1}$, and $\gamma_{2}$ are given in Table 1 and $e, f$, and $g$ are given in Table 2.

\subsection{First-order in- and out-of-phase concentration responses}

We impose small oscillations of the illumination at the angular frequency $\omega$. For the one-color system, we have

$$
I(t)=I^{0}[1+\varepsilon \sin (\omega t)]
$$

with $\varepsilon \ll 1$. In the two-color system, we propose to either modulate one of the two light intensities $I_{1}(t)$ and $I_{2}(t)$ or anticorrelate both of them at the same angular 
frequency

$$
\begin{aligned}
& I_{1}(t)=I_{1}^{0}\left[1+n_{1} \varepsilon \sin (\omega t)\right] \\
& I_{2}(t)=I_{2}^{0}\left[1+n_{2} \varepsilon \sin (\omega t)\right]
\end{aligned}
$$

where $\left(n_{1}, n_{2}\right)=(1,0)$ or $(0,1)$ for one modulated light intensity and $\left(n_{1}, n_{2}\right)=$ $(1,-1)$ for two modulated light intensities. The oscillations of illumination lead to oscillations of the photochemical rate constants

$$
\begin{aligned}
& k_{12}^{h \nu}(t)=k_{12}^{0}+\varepsilon k_{12}^{1} \sin (\omega t) \\
& k_{21}^{h \nu}(t)=k_{21}^{0}+\varepsilon k_{21}^{1} \sin (\omega t)
\end{aligned}
$$

For the one-color system, we have $k_{12}^{1}=k_{12}^{0}$ and $k_{21}^{1}=k_{21}^{0}$. For the two-color system, the expressions of $k_{12}^{1}$ and $k_{21}^{1}$ are obtained by introducing Eqs. (24,25) into Eqs. $(9,10)$. We look for solutions of Eqs. $(5,6)$ in the form of first-order expansions

$$
\begin{aligned}
& 1 B=1 B^{0}+\varepsilon 1 B^{1} \\
& 2 F=2 F^{0}+\varepsilon 2 F^{1},
\end{aligned}
$$

valid for sufficiently small oscillation amplitudes $(\varepsilon \ll 1)$. As previously, dimensionless variables and parameters are introduced. The dimensionless first-order amplitudes of concentration oscillation are given by

$$
\begin{aligned}
& \Psi_{1 B}^{1}=\frac{k_{+1}^{\Delta}}{k_{-1}^{\Delta}} 1 B^{1} \\
& \Psi_{2 F}^{1}=\frac{k_{+1}^{\Delta}}{k_{-1}^{\Delta}} 2 F^{1}
\end{aligned}
$$

In addition to the notations given in Table 1, we introduce three other dimensionless parameters in Table 3.

Under the hypothesis of small oscillations, Eqs. $(5,6)$ are linearized and written in matrix form

$$
\frac{d \mathcal{X}(\theta \delta)}{d \delta}=\mathcal{M} \mathcal{X}(\theta \delta)+\mathcal{F} \sin (\theta \delta)
$$




\begin{tabular}{|c|c|c|}
\hline & One-color system & Two-color system \\
\hline$\kappa$ & $\frac{k_{-1}^{\Delta}}{k_{\omega 1}^{\Delta}}$ & $\frac{k_{-1}^{\Delta}}{\sigma_{21,2} I_{2}^{0}}$ \\
$\theta$ & $\frac{\omega}{k_{21}}$ & $\frac{\sigma_{21,2} I_{2}^{0}}{\Delta}$ \\
$\delta$ & $k_{21}^{\Delta} t$ & $\sigma_{21,2} I_{2}^{0} t$ \\
\hline
\end{tabular}

Table 3: Expression of the dimensionless parameters $\kappa, \theta$, and $\delta$ for the one- and two-color systems.

\begin{tabular}{|c|c|c|}
\hline & One-color system & Two-color system \\
\hline$a$ & $\kappa\left(2 \Psi_{1 B}^{0}+\Psi_{2 F}^{0}-\left(\zeta_{R}+\zeta_{F}+1\right)\right)$ & $\kappa\left(2 \Psi_{1 B}^{0}+\Psi_{2 F}^{0}-\left(\zeta_{R}+\zeta_{F}+1\right)\right)$ \\
$b$ & $\kappa\left(\Psi_{1 B}^{0}-\zeta_{R}\right)$ & $\kappa\left(\Psi_{1 B}^{0}-\zeta_{R}\right)$ \\
$c$ & $-\eta$ & $-\gamma_{1} \eta+\gamma_{2}$ \\
$d$ & $-(\eta(\gamma+1)+1)$ & $-\left(\eta\left(\gamma_{1}+1\right)+\gamma_{2}+1\right)$ \\
$N$ & 1 & $\left|n_{1}-n_{2}\right|$ \\
$h$ & 1 & $\frac{\eta\left(\gamma_{1}-\gamma_{2}\right)}{\eta \gamma_{1}+\gamma_{2}}$ \\
\hline
\end{tabular}

Table 4: Expression of $a, b, c, d, N$, and $h$ for the one- and two-color systems, where $\kappa$ is given in Table 3, $\Psi_{1 B}^{0}$ and $\Psi_{2 F}^{0}$ in Eqs. $(19,20,22)$, and $\zeta_{R}, \zeta_{F}, \eta, \gamma, \gamma_{1}$, and $\gamma_{2}$ in Table 1.

where the vectors are

$$
\begin{aligned}
\mathcal{X} & =\left(\begin{array}{c}
\Psi_{1 B}^{1} \\
\Psi_{2 F}^{1}
\end{array}\right)=\mathcal{X}^{\cos } \cos (\theta \delta)+\mathcal{X}^{\sin } \sin (\theta \delta) \\
\mathcal{F} & =\left(\begin{array}{c}
0 \\
N h \Psi_{2 F}^{0}
\end{array}\right)
\end{aligned}
$$

and the matrix is

$$
\mathcal{M}=\left(\begin{array}{ll}
a & b \\
c & d
\end{array}\right)
$$

with $a, b, c, d, N$, and $h$ given in Table 4. Linearizing the dynamical equations in the limit of small forcing ensures that the analysis is generic: regardless of the specific nonlinearities of chemical kinetics, the following results can be applied. The linear system of equations for the dimensionless concentrations is easily solved in the eigenvector basis in which the equations are decoupled. The eigenvalues of $\mathcal{M}$ are given by

$$
\lambda_{ \pm}=\frac{a+d \pm \sqrt{(a-d)^{2}+4 b c}}{2}
$$


In the eigenvector basis, the dynamical state of the system is given by the vector

$$
\mathcal{Y}=\mathcal{P}^{-1} \mathcal{X}
$$

where $\mathcal{P}^{-1}$ is the inverse matrix of the change-of-basis matrix

$$
\mathcal{P}=\left(\begin{array}{cc}
\frac{b}{\sqrt{\left(\lambda_{+}-a\right)^{2}+b^{2}}} & \frac{b}{\sqrt{\left(\lambda_{-}-a\right)^{2}+b^{2}}} \\
\frac{\lambda_{+}-a}{\sqrt{\left(\lambda_{+}-a\right)^{2}+b^{2}}} & \frac{\lambda_{-} a}{\sqrt{\left(\lambda_{-}-a\right)^{2}+b^{2}}}
\end{array}\right)
$$

The coordinates of the vector $\mathcal{Y}=\mathcal{Y}^{\cos } \cos (\theta \delta)+\mathcal{Y}^{\sin } \sin (\theta \delta)$ obey the following decoupled equations

$$
\frac{d Y_{ \pm}(\theta \delta)}{d \delta}=\lambda_{ \pm} Y_{ \pm}(\theta \delta)+\alpha_{ \pm} \sin (\theta \delta)
$$

with

$$
\left(\begin{array}{c}
\alpha_{+} \\
\alpha_{-}
\end{array}\right)=\mathcal{P}^{-1} \mathcal{F}
$$

In the eigenvector basis, the solution is

$$
\begin{gathered}
Y_{ \pm}^{\cos }=-\frac{\theta \alpha_{ \pm}}{\theta^{2}+\lambda_{ \pm}^{2}} \\
Y_{ \pm}^{\sin }=-\frac{\lambda_{ \pm} \alpha_{ \pm}}{\theta^{2}+\lambda_{ \pm}^{2}}
\end{gathered}
$$

and in the initial basis, we find $\mathcal{X}=\mathcal{X}_{+}+\mathcal{X}_{-}$with $\mathcal{X}_{ \pm}=\mathcal{P} \mathcal{Y}_{ \pm}$. Bearing all above in mind, we obtain the expression of the dimensionless first-order amplitudes of concentration oscillations for $\mathbf{1 B}$ and $\mathbf{2 F}$

$$
\begin{aligned}
\Psi_{1 B}^{1, \cos } & =\frac{N \theta b(a+d) h \Psi_{2 F}^{0}}{\theta^{4}+\theta^{2}\left(a^{2}+d^{2}+2 b c\right)+(a d-b c)^{2}} \\
\Psi_{1 B}^{1, \sin } & =\frac{N b\left(a d-b c-\theta^{2}\right) h \Psi_{2 F}^{0}}{\theta^{4}+\theta^{2}\left(a^{2}+d^{2}+2 b c\right)+(a d-b c)^{2}} \\
\Psi_{2 F}^{1, \cos } & =-\frac{N \theta\left(\theta^{2}+a^{2}+b c\right) h \Psi_{2 F}^{0}}{\theta^{4}+\theta^{2}\left(a^{2}+d^{2}+2 b c\right)+(a d-b c)^{2}} \\
\Psi_{2 F}^{1, \sin } & =\frac{N\left[\theta^{2}(2 a+d)-a(a d-b c)\right] h \Psi_{2 F}^{0}}{\theta^{4}+\theta^{2}\left(a^{2}+d^{2}+2 b c\right)+(a d-b c)^{2}}
\end{aligned}
$$

where the parameters $a, b, c, d, N$, and $h$ are given in Table 4 , the dimensionless steady concentration $\Psi_{2 F}^{0}$ is given in Eq. (20), and the dimensionless frequency $\theta$ is given in Table 3. The expressions of the dimensionless first-order amplitudes for $\mathbf{1 F}$ and $\mathbf{R}$ are obtained using the law of conservation of matter given in Eqs. (3-4). 


\section{Analytical approach to the optimization of the out-of-phase concentration responses}

We wish to introduce a highly selective dynamic titration protocol discriminating the titrated species by the rate constants of their reaction with the photoswitchable reagent. We are concerned with identifying both the observable and the experimental conditions which are the most favorable to fulfill this goal.

The expressions (43-46) give access to the amplitudes of the in- and out-of-phase terms of the modulated concentrations. In this paper, we adopt the out-of-phase amplitude of the concentration oscillations to build the observable of a dynamic titration protocol. We focus on the behavior of $\mathbf{1 B}$ among the species involved in the reactions $(1,2)$. However, for the sake of completeness, we will also mention the remarkable properties of the species $\mathbf{1 F}$ and $\mathbf{2 F}$, which could be useful in similar systems. First of all, the out-of-phase amplitude of the concentration oscillations of $\mathbf{1 B}$ is proportional to the total concentration of the titrated species. Then, it is expected to present a band-pass filter behavior for species engaged in the set of reactions $(1,2)$, which should facilitate their discrimination. Indeed, species not affected by photochemical reactions do not respond to illumination modulation, whereas light fast-responding species respond in phase with the modulated illumination.

For the one-color system, the first-order amplitudes of the concentration oscillations are controlled by six dimensionless parameters $\eta, \gamma, \zeta_{R}, \zeta_{F}, \kappa$, and $\theta$, instead

of the nine initially introduced parameters, $\sigma_{12}, \sigma_{21}, I^{0}, R_{t o t}, F_{t o t}, k_{-1}^{\Delta}, \omega, k_{+1}^{\Delta}$, and $k_{21}^{\Delta}$. For the two-color system, the first-order amplitudes are controlled by seven dimensionless parameters $\eta, \gamma_{1}, \gamma_{2}, \zeta_{R}, \zeta_{F}, \kappa$, and $\theta$, instead of the eleven initially introduced parameters, $\sigma_{12,1}, \sigma_{21,1}, \sigma_{12,2}, \sigma_{21,2}, I_{1}^{0}, I_{2}^{0}, R_{t o t}, F_{t o t}, k_{-1}^{\Delta}, \omega$, and $k_{+1}^{\Delta}$.

It is essential to recognize the parameters which can be tuned in a titration protocol and the discriminative parameters which are specific to each targeted species. The scaled intensity of illumination, $\eta$, and the ratios of the isomerization cross- 
sections ( $\gamma$ for the one-color system or $\gamma_{1}$ and $\gamma_{2}$ associated with the light intensities $I_{1}$ and $I_{2}$ for the two-color system) characterize the kinetics of the reversibly photoswitchable reagent. The ratio of the complexation rate constant and photoswitching rate constant, $\kappa$, and the thermodynamic dissociation constant, $K_{d}=k_{-1}^{\Delta} / k_{+1}^{\Delta}$, are associated with the kinetics of the complexation of the targeted species. The parameters $\gamma$ or $\gamma_{1}$ and $\gamma_{2}$ are imposed by the photoswitchable reagent and $\kappa$ is set by the couple formed by the photoswitchable reagent and the titrated species. In contrast, the normalized concentration $\zeta_{R}$ of the targeted species, the normalized concentration $\zeta_{F}$ of the photoswitchable reagent, the scaled frequency $\theta$ of the forced oscillations, and the scaled intensity of illumination $\eta$ are monitored by the experimentalist and called control parameters.

\subsection{Optimization of $\Psi_{1 B}^{1, \text { cos }}$ with respect to the control param- eters}

In this subsection, we investigate whether the scaled out-of-phase amplitude of concentration oscillations $\Psi_{1 B}^{1, \text { cos }}$ exhibits extrema with respect to the control parameters. We determine analytical expressions of the control parameters associated with an extremum, further referred to as resonant parameter values labeled by an exponent $R$.

We first optimize $\Psi_{1 B}^{1, \text { cos }}$ with respect to the scaled frequency of the forced oscillations, $\theta$. Using Eq. (43), we find that $\Psi_{1 B}^{1, \text { cos }}$ exhibits a single optimum for a positive value of $\theta$ given by

$$
\theta^{R}=\sqrt{\frac{-\left(a^{2}+d^{2}+2 b c\right)+\sqrt{\left(a^{2}+d^{2}+2 b c\right)^{2}+12(a d-b c)^{2}}}{6}}
$$

Then, we consider optimizing $\Psi_{1 B}^{1, \text { cos }}\left(\theta^{R}\right)$ with respect to the scaled intensity of illumination, $\eta$. The exact expression of $\eta$ at the optimum could not be extracted in the general case, but it can be analytically retrieved in two experimentally relevant 
situations: (i) the complexation reaction (2) is much faster than the photoisomerization (1), i.e. $\kappa \gg 1$, which amounts to applying the quasi-steady state approximation to species $\mathbf{1 B}$, (ii) the reaction (1) is much faster than reaction (2), i.e. $\kappa \ll 1$, which amounts to applying the quasi-steady state approximation to species $\mathbf{2 F}$. To leading order, we find

$$
\begin{aligned}
& \theta^{R} \simeq\left|\frac{a d-b c}{a}\right|, \quad \kappa \gg 1 \\
& \theta^{R} \simeq\left|\frac{a d-b c}{d}\right|, \quad \kappa \ll 1
\end{aligned}
$$

Interestingly, both limit cases lead to the same value of the out-of-phase amplitude for $\theta=\theta^{R}$

$$
\Psi_{1 B}^{1, \cos }\left(\theta^{R}\right) \simeq \frac{N|b| \Psi_{2 F}^{0}}{2|a d-b c|}
$$

Although the expression does not depend on $\kappa$, it is nevertheless not valid for $\kappa \simeq$ 1. The expression of $\Psi_{1 B}^{1, \cos }\left(\theta^{R}\right)$ is still not sufficiently simplified to determine the resonant expression of $\eta$. Consequently, we further restrict our analysis to two concentration regimes, $\zeta_{R} \ll \zeta_{F}$ and $\zeta_{R} \gg \zeta_{F}$, leading to symmetrical expressions obtained by exchanging $\zeta_{R}$ and $\zeta_{F}$. In order to cover both regimes of approximation, we introduce $\zeta_{\max }$ and $\zeta_{\min }$ for $\sup \left(\zeta_{R}, \zeta_{F}\right)$ and $\inf \left(\zeta_{R}, \zeta_{F}\right)$, respectively, and obtain for the one-color system

$$
\Psi_{1 B}^{1, \cos }\left(\theta^{R}\right) \simeq \frac{N \eta \zeta_{\min } \zeta_{\max }}{2\left[\eta\left(\gamma\left(\zeta_{\max }+1\right)+1\right)+\zeta_{\max }+1\right]^{2}}
$$

We find a single optimum with respect to $\eta$ for

$$
\eta^{R} \simeq \frac{\zeta_{\max }+1}{\gamma\left(\zeta_{\max }+1\right)+1}
$$

and the out-of-phase amplitude optimized with respect to $\theta$ and $\eta$ is

$$
\Psi_{1 B}^{1, \cos }\left(\theta^{R}, \eta^{R}\right) \simeq \frac{N \zeta_{\min } \zeta_{\max }}{8\left(\gamma\left(\zeta_{\max }+1\right)+1\right)\left(\zeta_{\max }+1\right)}
$$


Similarly, we find for the two-color system

$$
\begin{aligned}
\Psi_{1 B}^{1, \cos }\left(\theta^{R}\right) & \simeq \frac{N\left(\gamma_{1}-\gamma_{2}\right) \eta \zeta_{\min } \zeta_{\max }}{2\left[\eta\left(\zeta_{\max }+\gamma_{1}+1\right)+\zeta_{\max }+\gamma_{2}+1\right]^{2}} \\
\eta^{R} & \simeq \frac{\zeta_{\max }+\gamma_{2}+1}{\zeta_{\max }+\gamma_{1}+1} \\
\Psi_{1 B}^{1, \cos }\left(\theta^{R}, \eta^{R}\right) & \simeq \frac{N\left(\gamma_{1}-\gamma_{2}\right) \zeta_{\min } \zeta_{\max }}{8\left(\zeta_{\max }+\gamma_{1}+1\right)\left(\zeta_{\max }+\gamma_{2}+1\right)}
\end{aligned}
$$

An optimum of $\Psi_{1 B}^{1, \cos }\left(\theta^{R}, \eta^{R}\right)$ with respect to the maximum $\zeta_{\max }$ can be found for the resonant value

$$
\zeta_{\max }^{R} \simeq \sqrt{\frac{\gamma+1}{\gamma}}
$$

leading to

$$
\Psi_{1 B}^{1, \cos }\left(\theta^{R}, \eta^{R}, \zeta_{\max }^{R}\right) \simeq \frac{N \zeta_{\min }}{8 \sqrt{\gamma}(2 \sqrt{\gamma(\gamma+1)}+2 \gamma+1)}
$$

For the two-color system, we derive

$$
\begin{aligned}
\zeta_{\max }^{R} & \simeq \sqrt{\left(\gamma_{1}+1\right)\left(\gamma_{2}+1\right)} \\
\Psi_{1 B}^{1, \cos }\left(\theta^{R}, \eta^{R}, \zeta_{\max }^{R}\right) & \simeq \frac{N\left(\gamma_{1}-\gamma_{2}\right) \zeta_{\min }}{8\left(\sqrt{\gamma_{1}+1}+\sqrt{\gamma_{2}+1}\right)^{2}}
\end{aligned}
$$

\subsection{Optimization of $\Psi_{1 F}^{1, \cos }$ and $\Psi_{2 F}^{1, \text { cos }}$ with respect to the con- trol parameters}

An analogous approach is adopted to find approximate expressions for the out-ofphase amplitudes $\Psi_{1 F}^{1, \cos }$ and $\Psi_{2 F}^{1, \text { cos }}$ of the modulation of $\mathbf{1 F}$ and $\mathbf{2 F}$ concentrations and the associated resonance conditions. Depending on the rates of complexation with respect to photoisomerization described by the $\kappa$ parameter, different behaviors are obtained. In the case of a slower complexation $(\kappa \ll 1)$, we find not one but three extrema in the $(\theta, \eta)$ parameter space. The resonant parameter values associated with a nonvanishing amplitude obey

$$
\theta^{R_{1}} \simeq|d|, \quad \theta^{R_{2}} \simeq\left|\frac{a d-b c}{d}\right|
$$


The first resonance $R_{1}$ is associated with the behavior of the photoisomerization subsystem given in Eq. (1). ${ }^{11,53,54}$ Indeed, in a two-state exchange, the resonant value of the angular frequency is equal to the inverse of the relaxation time of the reaction. Here, the dimensionless parameter $d$ corresponds to $k_{12}^{0}+k_{21}^{0}+k_{21}^{\Delta}$ for the scaled parameters. The second resonance $R_{2}$ associated with species $\mathbf{1 F}$ and $\mathbf{2 F}$ leads to the same condition (Eq. (49)) as for species 1B.

In the case of a slower photoisomerization $(\kappa \gg 1), \Psi_{1 F}^{1, \text { cos }}$, and $\Psi_{2 F}^{1, \text { cos }}$ exhibit a single extremum at

$$
\theta^{R} \simeq\left|\frac{a d-b c}{a}\right|
$$

which corresponds to the resonance condition associated with $\mathbf{1 B}$ given in Eq. (48).

\section{$5 \quad$ Numerical approach to the optimization of $1 B^{1, \cos }$ with respect to the control parameters}

\subsection{Behavior in the space of the control parameters}

The analytical calculations rely on approximations and provide partial knowledge of the behaviors of the out-of-phase amplitudes. Whereas the analytical $\theta^{R}$ expression is exact, the corresponding $\eta^{R}$ expression is not explicit when the rate constants associated with reactions $(1)$ and $(2)$ are comparable $(\kappa \simeq 1)$ or when the dimensionless concentrations $\zeta_{R}$ and $\zeta_{F}$ take similar values. In order to validate the approximations, we compare the analytical predictions with numerical solutions. We determine the parameter values associated with optima of the scaled out-of-phase amplitude.

Figure 1 displays the numerically computed dependence of the out-of-phase amplitude of the 1B concentration on the control parameters. Experimentally relevant values of $\gamma$ or $\gamma_{1}$ and $\gamma_{2}$ have been chosen. The $\mathbf{1 B}$ concentration is scaled by its upper value $\min \left(R_{t o t}, F_{\text {tot }}\right)$. Figures 1a,b evidence the predicted resonant behavior in the $(\theta, \eta)$ space and underline the relevance of the analytical resonant conditions. For the one-color system, the analytical predictions lead to $\left(\theta^{R}=0.69, \eta^{R}=0.92\right)$ 
a

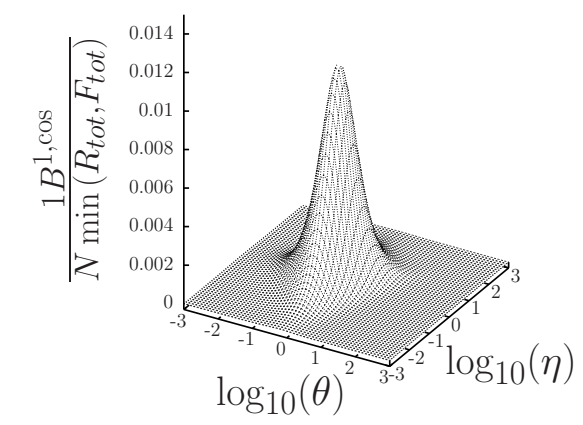

c

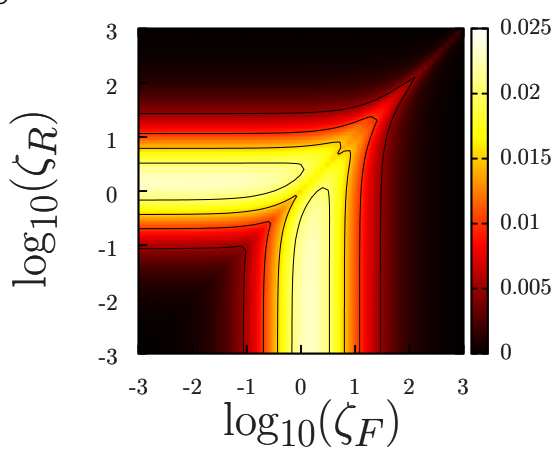

b

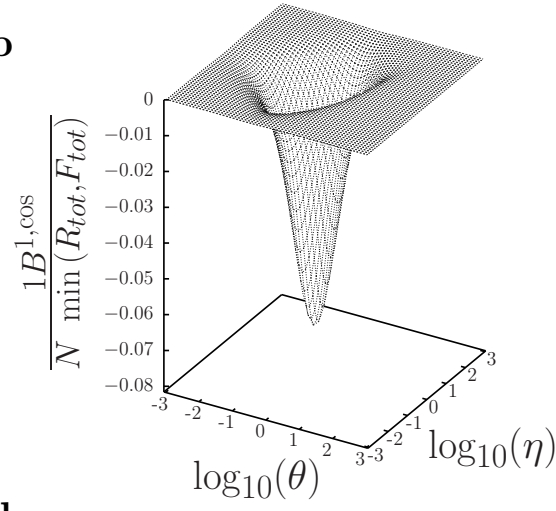

d

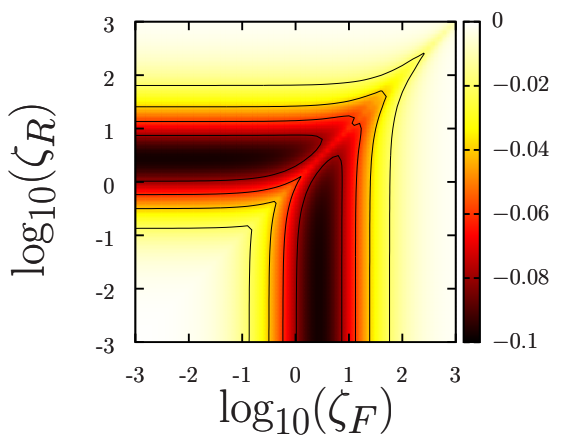

Figure 1: Numerically computed dependence of the scaled out-of-phase amplitude of the 1B concentration on the control parameters for the $(\mathrm{a}, \mathrm{c})$ one-color system $(\gamma=1)$ and $(\mathrm{b}, \mathrm{d})$ two-color system $\left(\gamma_{1}=1 / 5, \gamma_{2}=5\right)$. (a,b) Dependence of the normalized out-of-phase amplitude $1 B^{1, \cos } / N \min \left(R_{t o t}, F_{t o t}\right)$ on the dimensionless angular frequency $\theta$ and intensity of illumination $\eta$ for fixed values of $\zeta_{R}=0.1$ (dimensionless concentration of the titrated species), $\zeta_{F}=10$ (dimensionless concentration of the photoswitchable reagent), and $\kappa=0.1$ (ratio of complexation and isomerization rate constants). (c,d) Dependence of $1 B^{1, \cos }\left(\theta^{R}, \eta^{R}\right) / N \min \left(R_{t o t}, F_{t o t}\right)$ on $\zeta_{R}$ and $\zeta_{F}$, for a fixed value of $\kappa=0.1$.

while the numerical computations result in $\left(\theta^{R}=0.69, \eta^{R}=0.79\right)$. Similarly, for the two-color system, we analytically obtain $\left(\theta^{R}=0.41, \eta^{R}=1.43\right)$ and numerically find $\left(\theta^{R}=0.41, \eta^{R}=1.44\right)$. For a fixed value of $\zeta_{m i n}$, Figs. $1 \mathrm{c}, \mathrm{d}$ validate the presence of an optimum with respect to $\zeta_{\max }$ for both the one- and two-color systems. The observable needs to be proportional to $\zeta_{R}$ in order to be used in a titration protocol. Hence, $\zeta_{R}$ cannot be imposed by resonance conditions. In the following, we consider the approximation $\zeta_{F} \gg \zeta_{R}$ and the resonance with respect to $\zeta_{F}$. Figures 1c,d point out the relevance of the analytic expressions to locate the resonance. Specifically, for 
the one-color system and $\zeta_{R}=0.1$, we analytically find $\zeta_{F}^{R}=1.41$ while $\zeta_{F}^{R}=1.60$ is numerically computed. For the two-color system we analytically obtain $\zeta_{F}^{R}=2.63$ to be compared to the numerical value $\zeta_{F}^{R}=2.87$.

The resonant behavior in the $(\theta, \eta)$ space is robust. It is observed for all the values of $\gamma$ or $\gamma_{1}$ and $\gamma_{2}$ (e.g. for any type of photoswitching behavior of the photoswitchable reagent) and of $\kappa$ (e.g. for any ratio of the complexation rate constant and photoswitching rate constant). The resonance phenomenon in the $\left(\zeta_{R}, \zeta_{F}\right)$ space is robust as well. For all the investigated values of $\kappa$ and $\gamma, 1 B^{1, \cos }\left(\theta^{R}, \eta^{R}\right) / \min \left(R_{\text {tot }}, F_{\text {tot }}\right)$ exhibits a maximum $\zeta_{F}^{R}>1$ with respect to $\zeta_{F}$ when $\zeta_{R}<1$.

\subsection{Behavior with respect to the kinetically fixed parame- ters}

\subsubsection{One-color system}

We address the case of photoswitchable reagents whose isomerization is driven at one wavelength and study the impact of the parameters $\gamma$ and $\kappa$ on the resonance of the out-of-phase amplitude $1 B^{1, \text { cos }}$.

We first examine the significance of the photoswitching features expressed by $\gamma$ on the position of the resonance of $1 B^{1, \cos }$ in the $(\theta, \eta)$ space (see Fig. 1a). As shown in Fig. 2a, $\theta^{R}$ does not significantly depend on $\gamma$. In contrast, $\eta^{R}$ strongly depends on $\gamma$ (Fig. 2b). In particular, for large $\gamma, \eta^{R}$ is proportional to $1 / \gamma$. Then, we analyze the role of $\gamma$ on the resonance of $1 B^{1, \text { cos }}$ in $\zeta_{F}$ for fixed $\zeta_{R}<1$ (Fig. 2c). For small $\gamma, \zeta_{F}^{R}$ is proportional to $1 / \sqrt{\gamma}$ but converges to 1 for large $\gamma$. In Figs. SI 1a,b,c of the Supplementary Information, we report on the deviations between the analytically and numerically computed values of $\theta^{R}, \eta^{R}$, and $\zeta_{F}^{R}$, which originate from the expansion used to derive Eqs. $(52,57)$. As shown in Fig. SI 1a of Supplementary Information, the introduction of the approximate values of $\eta^{R}$ and $\zeta_{F}^{R}$ into the exact expression of $\theta^{R}$ given in Eq. (47) induces a difference between the values retrieved from analytical and numerical computations. It is worth noting 
that the observed deviations are small compared to the width of the resonance peak where the amplitude drops to half of the resonance value. As a result, the analytical predictions are fully exploitable.

a

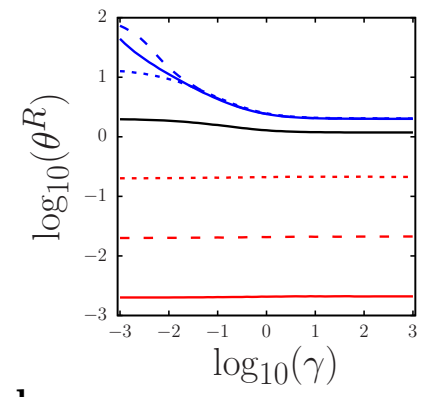

d

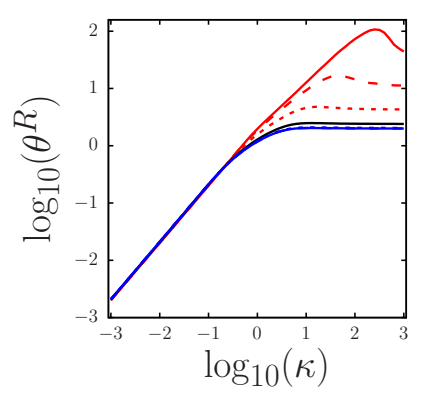

b

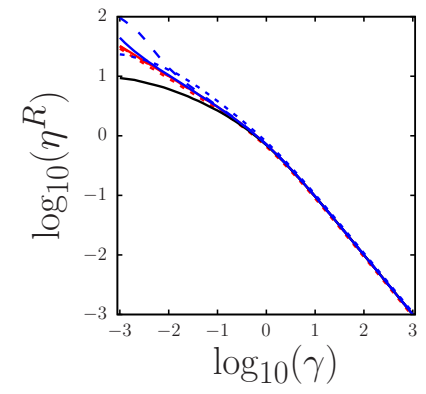

e

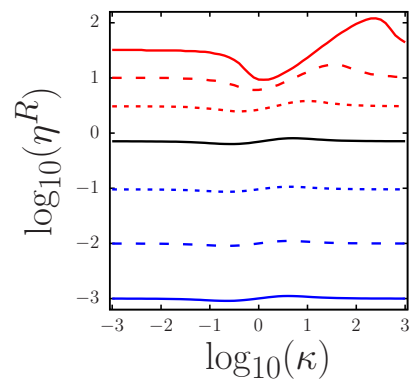

c

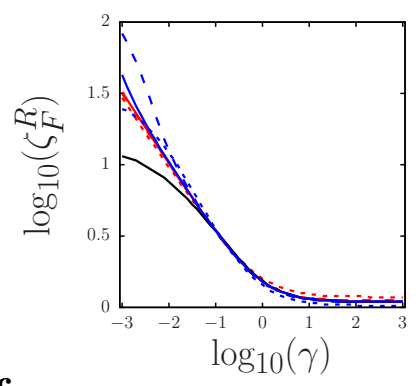

f

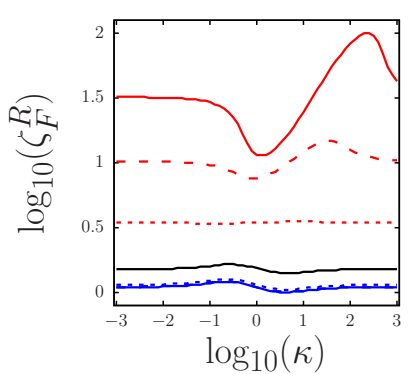

Figure 2: One-color system. Dependence of the resonant values of the control parameters on $\gamma$ (ratio of backward and forward photochemical isomerization rate constants) and $\kappa$ (ratio of complexation and isomerization rate constants). Dependence of (a) $\theta^{R}$, (b) $\eta^{R}$, (c) $\zeta_{F}^{R}$ on $\gamma$ for several fixed values of $\kappa$ equal to $10^{-3}$ (red solid line), $10^{-2}$ (red dashed line), $10^{-1}$ (red dotted line), 1 (black solid line), $10^{1}$ (blue dotted line), $10^{2}$ (blue dashed line), $10^{3}$ (blue solid line). (d,e,f) same caption as in $(\mathrm{a}, \mathrm{b}, \mathrm{c})$ by exchanging $\gamma$ and $\kappa$. The numerical computations are performed for a fixed value of $\zeta_{R}=0.1$ (dimensionless concentration of the titrated species).

Finally, we study the impact of $\gamma$ on the amplitude of the resonance of $1 B^{1, \cos }\left(\theta^{R}, \eta^{R}, \zeta_{F}^{R}\right)$. Figure 3a and Eq. (58) show that $1 B^{1, \cos }\left(\theta^{R}, \eta^{R}, \zeta_{F}^{R}\right) / \min \left(R_{t o t}, F_{t o t}\right)$ is a decreasing function of $\gamma$ and essentially vanishes for $\gamma>1$. The maximal $1 B^{1, \text { cos }}$ value reaches $14 \%$ of $\min \left(R_{t o t}, F_{t o t}\right)$ and $25 \%$ of the steady state value $1 B^{0}$.

We examine the effect of the complexation kinetics expressed by the parameter $\kappa$ on the resonance. As shown in Fig. $2 \mathrm{~d}, \theta^{R}$ is proportional to $\kappa$ at small $\kappa$ values, whereas it does not significantly depend on $\kappa$ at large $\kappa$. This observation 
$\mathbf{a}$

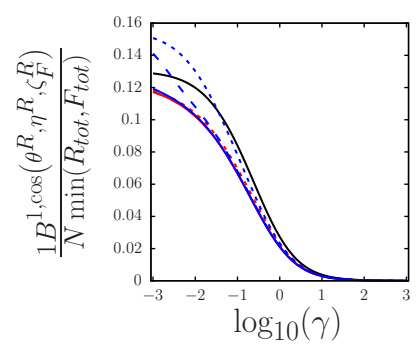

b

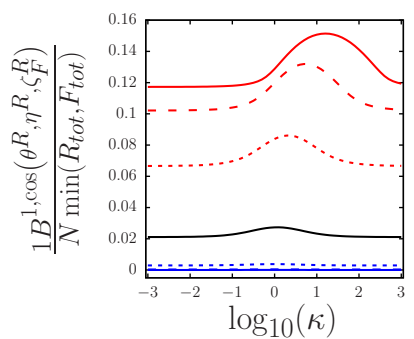

Figure 3: One-color system. (a) Dependence of the resonant out-of-phase amplitude of oscillations of concentration $1 B^{1, \cos }\left(\theta^{R}, \eta^{R}, \zeta_{F}^{R}\right) / N \min \left(R_{t o t}, F_{t o t}\right)$ on $\gamma$ for $\zeta_{R}=$ 0.1 and for several fixed values of $\kappa$ equal to $10^{-3}$ (red solid line), $10^{-2}$ (red dashed line), $10^{-1}$ (red dotted line), 1 (black solid line), $10^{1}$ (blue dotted line), $10^{2}$ (blue dashed line), $10^{3}$ (blue solid line). (b) same caption as in (a) by exchanging $\gamma$ and $\kappa$.

was expected from Eqs. (48,49). As anticipated from Eqs. $(52,57), \eta^{R}$ and $\zeta_{F}^{R}$ are essentially independent of $\kappa$ (Figs. 2e,f). However, a second-order dependence of $\zeta_{F}^{R}$ on $\kappa$ is numerically observed when $\gamma \ll 1$. Figures SI 1d,e,f of the Supplementary Information show that the deviations between the analytically and numerically computed values of $\theta^{R}, \eta^{R}$ and $\zeta_{F}^{R}$ can be neglected.

The out-of-phase amplitude $1 B^{1, \cos }\left(\theta^{R}, \eta^{R}, \zeta_{F}^{R}\right) / \min \left(R_{t o t}, F_{t o t}\right)$ does not significantly depend on $\kappa$ as illustrated in Fig. 3b and Eq. (58).

\subsubsection{Two-color system}

For a two-color system, the kinetic parameters to be considered are $\gamma_{1}, \gamma_{2}$, and $\kappa$. The choice of the dimensionless parameters has introduced a symmetry breaking in a system initially symmetric with respect to the permutations between the light intensities $I_{1}^{0}$ and $I_{2}^{0}$ and the isomerization cross-sections $\left(\sigma_{12,1}, \sigma_{21,1}\right)$ and $\left(\sigma_{12,2}, \sigma_{21,2}\right)$. It is worth noting that the sign of the amplitude $1 B^{1, \cos }$ is imposed by the sign of $\gamma_{1}-\gamma_{2}$. We discuss the results in the case $\gamma_{1}<\gamma_{2}$ leading to a negative amplitude.

We study the impact of the ratios $\gamma_{1}$ and $\gamma_{2}$ of the isomerization cross-sections corresponding to the light intensities $I_{1}^{0}$ and $I_{2}^{0}$ on the position of the resonance 
$1 B^{1, \mathrm{cos}}$ in the control parameter space. Analytical expressions given in Eqs. $(47,55,59)$ and numerical results both lead to the following conclusions. For $\kappa \gg 1, \theta^{R}$ is an increasing function of $\gamma_{1}$ and $\gamma_{2}$, whereas $\theta^{R}$ does not depend on $\gamma_{1}$ and $\gamma_{2}$ for $\kappa \ll 1$. The resonant value $\eta^{R}$ is a decreasing function of $\gamma_{1}$ and an increasing function of $\gamma_{2}$. We find that $\zeta_{F}^{R}$ is an increasing function of $\gamma_{1}$ and $\gamma_{2}$. Figures 4a,b,c show the numerical results in the particular case $\gamma_{2}=1 / \gamma_{1}$ which corresponds to reasonable experimental conditions. The comparison between the analytical predictions and the numerical results is made precise in Figs. SI 2a,b,c of the Supplementary Information for $\gamma_{2}=1 / \gamma_{1}$ The analytical results can be used with a satisfying accuracy in a wide parameter range.

The impact of $\gamma_{1}$ and $\gamma_{2}$ on the resonance of $1 B^{1, \cos }\left(\theta^{R}, \eta^{R}, \zeta_{F}^{R}\right)$ is shown in Fig. 5a in the particular case $\gamma_{2}=1 / \gamma_{1}$. The amplitude is an increasing function of $\left|\gamma_{1}-\gamma_{2}\right|$ and reaches a plateau for $\left|\gamma_{1}-\gamma_{2}\right| \gg 100$, according to Eq. (60). The largest $\left|1 B^{1, \cos }\right|$ reaches $12.5 \%$ of $\min \left(R_{t o t}, F_{t o t}\right)$ for a single modulated light intensity and $25 \%$ of $\min \left(R_{t o t}, F_{t o t}\right)$ for two modulated light intensities. Similarly, | $1 B^{1, \cos } \mid$ reaches $25 \%$ of the steady state value $1 B^{0}$ for a single modulated light intensity and $50 \%$ of the steady state value $1 B^{0}$ for two modulated light intensities.

The dependences of $\theta^{R}, \eta^{R}$, and $\zeta_{F}^{R}$ on $\kappa$ are similar to the ones that have already been discussed in a one-color system. Figures 4a,b,c display the numerical results for $\gamma_{2}=1 / \gamma_{1}$. Figures SI 2d,e,f of the Supplementary Information show that the discrepancies between the analytically and numerically computed values of $\theta^{R}, \eta^{R}$, and $\zeta_{F}^{R}$ are small.

The amplitude $1 B^{1, \cos }\left(\theta^{R}, \eta^{R}, \zeta_{F}^{R}\right)$ is nearly independent of $\kappa$, as displayed in Fig. $5 \mathrm{~b}$ and in Eq. (60).

The behaviors of the one- and two-color systems are similar. However for a twocolor system with two modulated light intensities, the optimized amplitude can be 
a

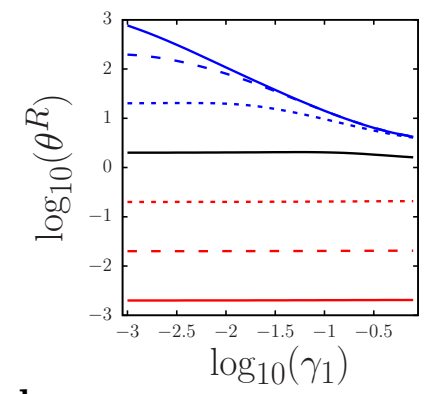

d

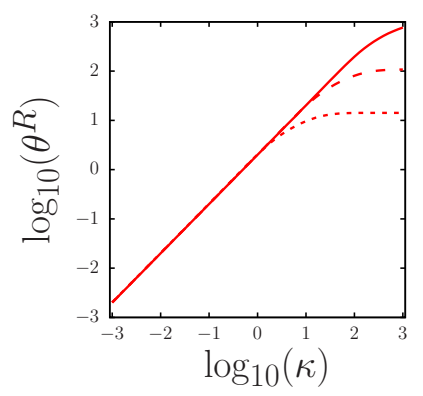

b

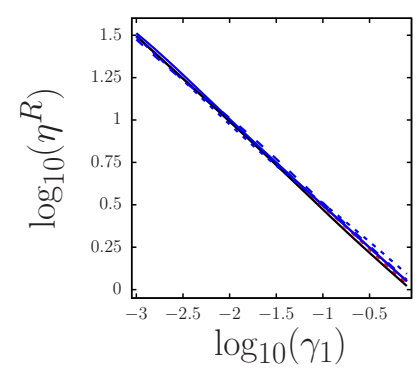

e

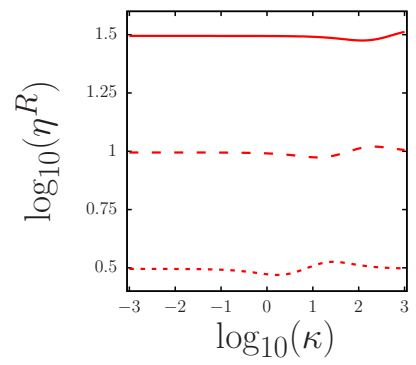

C

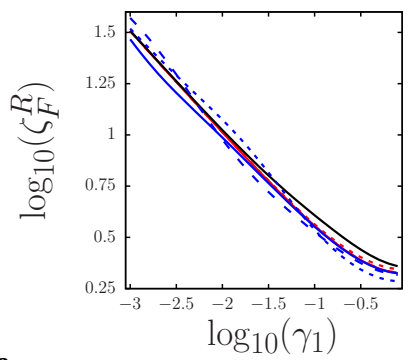

f

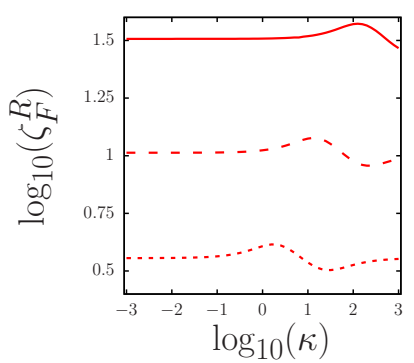

Figure 4: Two-color system. Dependence of the resonant values of the control parameters on $\gamma_{1}$ (ratio of forward and backward photochemical isomerization rate constants corresponding to the light intensity $I_{1}^{0}$ ) and $\kappa$ (ratio of complexation and isomerization rate constants). Dependence of (a) $\theta^{R}$, (b) $\eta^{R}$, (c) $\zeta_{F}^{R}$ on $\gamma_{1}$ for several fixed values of $\kappa$ equal to $10^{-3}$ (red solid line), $10^{-2}$ (red dashed line), $10^{-1}$ (red dotted line), 1 (black solid line), $10^{1}$ (blue dotted line), $10^{2}$ (blue dashed line), $10^{3}$ (blue solid line). Dependence of (d) $\theta^{R}$, (e) $\eta^{R}$, (f) $\zeta_{F}^{R}$ on $\kappa$ for several fixed values of $\gamma_{1}$ equal to $10^{-3}$ (red solid line), $10^{-2}$ (red dashed line), $10^{-1}$ (red dotted line). The numerical computations are performed for a fixed value of $\zeta_{R}=0.1$ (dimensionless concentration of the titrated species) and with variable $\gamma_{2}=1 / \gamma_{1}$.

doubled with respect to the one of the one-color system.

\section{Protocol for light-assisted dynamic titration}

The preceding results can be used to propose a highly selective dynamic titration protocol, able to discriminate components $\mathbf{R}$ in a mixture by the rate constants $k_{+1}^{\Delta}$ and $k_{-1}^{\Delta}$ of their reaction with the photoswitchable reagent, viewed as the titrating reagent. Focussing on a targeted component, we suggest to tune the control parameters and maximize the amplitude of the out-of-phase response of its associated bound state $1 \mathrm{~B}$. Indeed, resonant or optimized values of the parameters $\zeta_{R}, \zeta_{F}, \theta$, 
$\mathbf{a}$

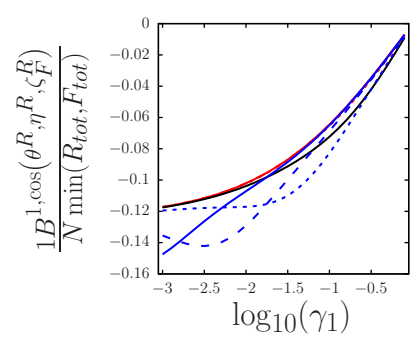

b

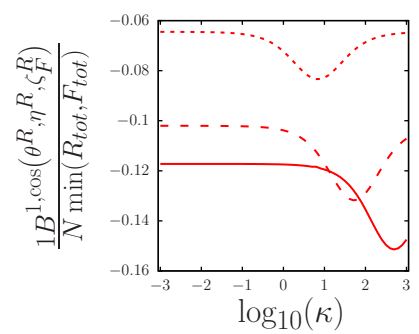

Figure 5: Two-color system. (a) Dependence of the resonant out-of-phase amplitude of oscillation of concentration $1 B^{1, \cos }\left(\theta^{R}, \eta^{R}, \zeta_{F}^{R}\right) / N \min \left(R_{t o t}, F_{t o t}\right)$ on $\gamma_{1}$ for several fixed values of $\kappa$ equal to $10^{-3}$ (red solid line), $10^{-2}$ (red dashed line), $10^{-1}$ (red dotted line), 1 (black solid line), $10^{1}$ (blue dotted line), $10^{2}$ (blue dashed line), $10^{3}$ (blue solid line). (b) Dependence of the resonant out-of-phase amplitude on $\kappa$ for several fixed values of $\gamma_{1}$ equal to $10^{-3}$ (red solid line), $10^{-2}$ (red dashed line), $10^{-1}$ (red dotted line). The numerical computations are performed for a fixed value of $\zeta_{R}=0.1$ and with variable $\gamma_{2}=1 / \gamma_{1}$.

and $\eta$ provide a significant observable for $\mathbf{1 B}$ proportional to the total concentration $R_{t o t}$ of the targeted species while eliminating the contributions from the other mixture components. According to Tables 1 and 3 , choosing $\zeta_{R}, \zeta_{F}, \theta$, and $\eta$ imposes constraints on the concentrations $R_{t o t}$ and $F_{t o t}$ of the targeted component and the titrating reagent, the angular frequency $\omega$ of the forced illumination oscillations, and the illumination intensities $I^{0}$ in a one-color system and $I_{1}^{0}$ and $I_{2}^{0}$ in a two-color system. In a user-friendly approach, we derive the optimized expressions of the dimensioned control parameters and design an experimental titrating protocol.

\subsection{Setting the concentrations of the targeted component and the titrating reagent}

We assume that the total concentration $R_{t o t}$ of the target cannot be tuned but is significantly lower than the tunable total concentration of the photoswitchable reagent $F_{\text {tot }}$. We further assume that $R_{\text {tot }} \ll K_{d}$ which leads to simpler expressions of the illumination control parameters presented in the following.

We use Eqs. $(57,59)$ to relate the resonant value $F_{\text {tot }}^{R}$ to the dimensioned pa- 
rameters of the targeted component. We obtain for the one-color system

$$
F_{t o t}^{R} \simeq K_{d} \sqrt{1+\frac{\sigma_{12}}{\sigma_{21}}}
$$

and for the two-color system

$$
F_{t o t}^{R} \simeq K_{d} \sqrt{\left(1+\frac{\sigma_{12,1}}{\sigma_{21,1}}\right)\left(1+\frac{\sigma_{12,2}}{\sigma_{21,2}}\right)}
$$

where $\left(\sigma_{12}, \sigma_{21}\right)$ and $\left(\sigma_{12,1}, \sigma_{21,1}, \sigma_{12,2}, \sigma_{21,2}\right)$ are specific to the photoswitchable reagent and $K_{d}=k_{-1}^{\Delta} / k_{+1}^{\Delta}$ is the thermodynamic dissociation constant specific to the couple formed by the photoswitchable reagent and the targeted component.

\subsection{Setting the illumination parameters}

The control parameters associated with illumination are the frequency $\omega$ and the light intensities, $I^{0}$ in a one-color system and $I_{1}^{0}$ and $I_{2}^{0}$ in a two-color system.

\subsubsection{One-color system}

Using Eqs. $(48,49,52)$, valid in the approximation $R_{t o t} \ll F_{\text {tot }}$, we find the following resonant values for the one-color system

$$
\begin{aligned}
& \omega^{R} \simeq k_{21}^{\Delta}\left(1+\sqrt{1+\frac{\sigma_{12}}{\sigma_{21}}}\right), \text { for } k_{-1}^{\Delta} \gg k_{21}^{\Delta} \\
& \omega^{R} \simeq 2 k_{-1}^{\Delta}, \quad \text { for } k_{-1}^{\Delta} \ll k_{21}^{\Delta} \\
& I^{0, R} \simeq \frac{k_{21}^{\Delta}}{\sqrt{\sigma_{21}\left(\sigma_{12}+\sigma_{21}\right)}}
\end{aligned}
$$

Only Eq. (66) links the resonant value of the angular frequency with the specific kinetics of the target. The other resonance conditions on the illumination parameters only involve the kinetics of the titrating reagent, which is common to all the titrated species. Extracted from Eq. (58), the optimized amplitude is given by

$$
1 B^{1, \cos }\left(\omega^{R}, I^{0, R}, F_{t o t}^{R}\right) \simeq \frac{R_{t o t}}{8 \sqrt{\frac{\sigma_{21}}{\sigma_{12}}}\left(2 \sqrt{\frac{\sigma_{21}}{\sigma_{12}}\left(\frac{\sigma_{21}}{\sigma_{12}}+1\right)}+2 \frac{\sigma_{21}}{\sigma_{12}}+1\right)}
$$




\subsubsection{Two-color system}

In a two-color system, we use the resonant values of the dimensionless parameters given in Eqs. $(48,49,55)$, valid in the approximation $R_{t o t} \ll F_{t o t}$ and obtain

$$
\begin{aligned}
\omega^{R} \simeq & \sqrt{\sigma_{21,2}\left(\sigma_{12,2}+\sigma_{21,2}\right)}\left(\sqrt{1+\frac{\sigma_{12,1}}{\sigma_{21,1}}}\right. \\
& \left.+\sqrt{1+\frac{\sigma_{12,2}}{\sigma_{21,2}}}\right) I_{2}^{0}, \quad \text { for } k_{-1}^{\Delta} \gg \sigma_{21,2} I_{2}^{0} \\
\omega^{R} \simeq & 2 k_{-1}^{\Delta}, \quad \text { for } k_{-1}^{\Delta} \ll \sigma_{21,2} I_{2}^{0} \\
\left(\frac{I_{1}^{0}}{I_{2}^{0}}\right)^{R} \simeq & \sqrt{\frac{\left(\sigma_{12,2}+\sigma_{21,2}\right) \sigma_{21,2}}{\left(\sigma_{12,1}+\sigma_{21,1}\right) \sigma_{21,1}}}
\end{aligned}
$$

As for the one-color system, only the resonant value of the angular frequency is related to the kinetics of the target according to Eq. (70). The optimized amplitude is deduced from Eq. (60)

$$
1 B^{1, \cos }\left(\omega^{R},\left(I_{1}^{0} / I_{2}^{0}\right)^{R}, F_{t o t}^{R}\right) \simeq \frac{N R_{t o t}\left(\frac{\sigma_{12,1}}{\sigma_{21,1}}-\frac{\sigma_{12,2}}{\sigma_{21,2}}\right)}{8\left(\sqrt{\frac{\sigma_{12,1}}{\sigma_{21,1}}+1}+\sqrt{\frac{\sigma_{12,2}}{\sigma_{21,2}}+1}\right)^{2}}
$$

Choosing to scale the light intensity $I_{1}^{0}$ and the angular frequency $\omega$ by the light intensity $I_{2}^{0}$ is arbitrary, the roles of $I_{1}^{0}$ and $I_{2}^{0}$ being symmetrical. Interestingly, optimization of the illumination parameters imposes two conditions whereas the experimentalist has three degrees of freedom, $I_{1}^{0}, I_{2}^{0}$, and $\omega$. The method is therefore very flexible. The resonance condition on the illumination intensities does not independently set $I_{1}^{0}$ and $I_{2}^{0}$, but sets the ratio $\left(\frac{I_{1}^{0}}{I_{2}^{0}}\right)^{R}$. In the case of fast complexation compared to isomerization, increasing the illumination intensities leads to increasing the angular frequency, whereas the angular frequency remains unchanged otherwise. In addition, the choice of $I_{2}^{0}$ determines the rate-limiting step: Small $I_{2}^{0}$ values lead to slower photoisomerization and large $I_{2}^{0}$ values lead to slower complexation.

Comparing $k_{-1}^{\Delta}$ to $k_{21}^{\Delta}$ in the case of the one-color system and to $\sigma_{21,2} I_{2}^{0}$ in the case of the two-color system amounts to comparing the relaxation times of photoisomerization (reaction (1)) and complexation (reaction (2)). The expressions of 
the resonant angular frequency given in Eqs. $(65,69)$ are obtained when photoisomerization is the rate-limiting step. Conversely, Eqs. $(66,70)$ are obtained when complexation is the rate-determining step. Equations $(65,66)$ for the one-color system can be written

$$
\begin{aligned}
& \omega^{R} \simeq k_{21}^{\Delta}+I^{0, R}\left(\sigma_{12}+\sigma_{21}\right), \quad \text { for } \quad k_{-1}^{\Delta} \gg k_{21}^{\Delta} \\
& \omega^{R} \simeq k_{+1}^{\Delta} 1 F^{0}\left(I^{0, R}, F_{t o t}^{R}\right)+k_{-1}^{\Delta}, \quad \text { for } \quad k_{-1}^{\Delta} \ll k_{21}^{\Delta}
\end{aligned}
$$

and Eqs. $(65,66)$ for the two-color system lead to

$$
\begin{aligned}
& \omega^{R} \simeq\left(\left(\sigma_{12,1}+\sigma_{21,1}\right)\left(\frac{I_{1}^{0}}{I_{2}^{0}}\right)^{R}+\left(\sigma_{12,2}+\sigma_{21,2}\right)\right) I_{2}^{0}, \text { for } k_{-1}^{\Delta} \gg \sigma_{21,2} I_{2}^{0} \\
& \omega^{R} \simeq k_{+1}^{\Delta} 1 F^{0}\left(\left(I_{1}^{0} / I_{2}^{0}\right)^{R}, F_{t o t}^{R}\right)+k_{-1}^{\Delta}, \quad \text { for } \quad k_{-1}^{\Delta} \ll \sigma_{21,2} I_{2}^{0}
\end{aligned}
$$

Hence, in the two limit cases, the resonant angular frequency is equal to the inverse of the relaxation time of the rate-limiting step, exactly as in a chemical scheme with a single step. ${ }^{11,53,54}$ Moreover, when complexation is slower than isomerization, the value of the angular frequency, tuned to discriminate the targeted component, is the same in the two-color system (Eq. (70)) as in the one-color system (Eq. (66)).

It is worth noting that the resonance conditions setting light intensity $I^{0, R}$ or $\left(I_{1}^{0} / I_{2}^{0}\right)^{R}$ according to Eqs. $(67,71)$ impose

$$
k_{12}^{0}\left(I^{0, R}\right) 1 F^{0}\left(I^{0, R}, F_{\text {tot }}^{R}\right) \simeq k_{21}^{0}\left(I^{0, R}\right) 2 F^{0}\left(I^{0, R}, F_{\text {tot }}^{R}\right)
$$

for the one-color system and

$$
k_{12}^{0}\left(\left(I_{1}^{0} / I_{2}^{0}\right)^{R}\right) 1 F^{0}\left(\left(I_{1}^{0} / I_{2}^{0}\right)^{R}, F_{t o t}^{R}\right) \simeq k_{21}^{0}\left(\left(I_{1}^{0} / I_{2}^{0}\right)^{R}\right) 2 F^{0}\left(\left(I_{1}^{0} / I_{2}^{0}\right)^{R}, F_{t o t}^{R}\right)
$$

for the two-color system, where the rate constants are given in Eqs. (11-14) and the steady concentrations in Eqs. $(19,20,22)$ for resonant values of light intensity and total concentration of the photoswitchable reagent. Hence, the photoisomerization 
reaction is at equilibrium when the control parameters are resonant. Similarly, the resonance conditions setting the concentration $F_{t o t}^{R}$ of the photoswitchable reagent according to Eqs. $(63,64)$ lead to

$$
\begin{aligned}
& 1 F^{0}\left(I^{0, R}, F_{\text {tot }}^{R}\right) \simeq K_{d} \\
& 1 B^{0}\left(I^{0, R}, F_{\text {tot }}^{R}\right) \simeq R^{0}\left(I^{0, R}, F_{\text {tot }}^{R}\right) \simeq R_{\text {tot }} / 2
\end{aligned}
$$

for the one-color system and

$$
\begin{aligned}
& 1 F^{0}\left(\left(I_{1}^{0} / I_{2}^{0}\right)^{R}, F_{t o t}^{R}\right) \simeq K_{d} \\
& 1 B^{0}\left(\left(I_{1}^{0} / I_{2}^{0}\right)^{R}, F_{\text {tot }}^{R}\right) \simeq R^{0}\left(\left(I_{1}^{0} / I_{2}^{0}\right)^{R}, F_{\text {tot }}^{R}\right) \simeq R_{t o t} / 2
\end{aligned}
$$

for the two color system. Thus, the complexation reaction is at equilibrium for resonant values of the control parameters.

In summary, the optimized conditions bring out specific links between the kinetics of the target and the control parameters. From Eqs. $(63,67)$ and Eqs. $(64,71)$, it comes out that both the unbinding rate constant $k_{-1}^{\Delta}$ and the thermodynamic dissociation constant $K_{d}=k_{-1}^{\Delta} / k_{+1}^{\Delta}$ are discriminating parameters for both the one-

and two-color systems. In both cases, $k_{-1}^{\Delta}$ only affects the choice of the angular frequency $\omega^{R}$ while $K_{d}$ affects the choice of the concentration $F_{\text {tot }}^{R}$ of the titrating reagent. From Eqs. $(68,72)$, the optimized out-of-phase is proportional to the total concentration of the titrated species $R_{t o t}$.

\subsection{The light-assisted dynamic titration in action}

In view of the preceding results, we design a protocol relevant to discriminate a targeted species $\mathbf{R}$ against interfering compounds. The choice of the titrating species sets the photoisomerization cross-sections and the thermal isomerization rate constant. For both the one- and two-color systems, we propose a titrating protocol using $k_{+1}^{\Delta}$ and $k_{-1}^{\Delta}$ as discriminating parameters, for a given choice of the titrating reagent. The total concentration of the titrating species $F_{\text {tot }}^{R}$ is set according to Eqs. 
$(63,64)$ : the ratio of rate constants, $K_{d}=k_{-1}^{\Delta} / k_{+1}^{\Delta}$, of the targeted species specifies the total concentration $F_{\text {tot }}^{R}$. The angular frequency $\omega^{R}$ is set according to either Eqs. $(65,69)$ or Eqs. $(66,70)$, depending on the value of the unbinding rate constant $k_{-1}^{\Delta}$. The light intensity $I^{0, R}$ or the ratio $\left(I_{1}^{0} / I_{2}^{0}\right)^{R}$ is set to the resonant value given in Eqs. $(67,71)$.

Figure 6a for the one-color system and Fig. 6b for the two-color system display isodensity curves associated with the out-of-phase amplitude of $\mathbf{1 B}$ for three targeted species with different unbinding rate constants $k_{-1}^{\Delta}$ in the $\left(\omega, I^{0}\right)$ space and the $\left(\omega, I_{1}^{0} / I_{2}^{0}\right)$ space, respectively. For targeted species with different $k_{-1}^{\Delta}$ but the same value of the dissociation constant $K_{d}$, the position of the resonance with respect to $I^{0}$ and $I_{1}^{0} / I_{2}^{0}$ is the same for all the three targeted species, whereas the position of the resonance with respect to $\omega$ varies with the unbinding rate constant of the targeted species. Kinetic discrimination is efficient provided that $k_{-1}^{\Delta}<k_{21}^{\Delta}$ for the one-color system and $k_{-1}^{\Delta}<\sigma_{21,2} I_{2}^{0}$ for the two-color system. Hence, the choice of the titrating agent has to be adapted to the targeted species for dynamic titration to be successful.

Figure $6 \mathrm{c}$ for the one-color system and Fig. 6d for the two-color system show how the isodensity curves associated with the observable move in the $\left(F_{t o t}, I^{0}\right)$ space and the $\left(F_{t o t}, I_{1}^{0} / I_{2}^{0}\right)$ space, respectively, when the thermodynamic dissociation constant $K_{d}$ of the targeted species changes. For targeted species with different values of the dissociation constant but the same value of the unbinding rate constant $k_{-1}^{\Delta}$, the position of the resonance with respect to $I^{0}$ or $I_{1}^{0} / I_{2}^{0}$ is the same for all $K_{d}$ values. On the contrary, the position of the resonance with respect to $F_{t o t}$ significantly varies for the investigated values of $K_{d}$. Hence, kinetic discrimination for targets with different $k_{+1}^{\Delta}$ values will be efficient. 


\begin{tabular}{|c|c|}
\hline One-color system & Two-color system \\
\hline \multicolumn{2}{|c|}{ Fixed parameters associated with the titrating reagent } \\
\hline $\begin{array}{c}\sigma_{12}, \sigma_{21} \\
k_{21}^{\Delta} \\
\end{array}$ & $\begin{array}{c}\sigma_{12,1}, \sigma_{12,2}, \sigma_{21,1}, \sigma_{21,2} \\
k_{21}^{\Delta} \text { negligible } \\
\end{array}$ \\
\hline & Illumination modulation \\
\hline$I(t)=I^{0}[1+\varepsilon \sin (\omega t)]$ & $\begin{aligned} I_{1}(t) & =I_{1}^{0}\left[1+n_{1} \varepsilon \sin (\omega t)\right] \\
I_{2}(t) & =I_{2}^{0}\left[1+n_{2} \varepsilon \sin (\omega t)\right]\end{aligned}$ \\
\hline & $\begin{array}{c}\text { Total concentrations } \\
R_{t o t} \ll K_{d}\end{array}$ \\
\hline$F_{t o t}^{R} \simeq K_{d} \sqrt{\frac{\sigma_{12}+\sigma_{21}}{\sigma_{21}}}$ & $F_{t o t}^{R} \simeq K_{d} \sqrt{\left(1+\frac{\sigma_{12,1}}{\sigma_{21,1}}\right)\left(1+\frac{\sigma_{12,2}}{\sigma_{21,2}}\right)}$ \\
\hline $\begin{aligned} & I^{0, R} \simeq \frac{k_{21}^{\Delta}}{\sqrt{\sigma_{21}\left(\sigma_{12}+\sigma_{21}\right)}} \\
& \text { if } k_{-1}^{\Delta} \gg k_{21}^{\Delta}, \omega^{R} \simeq k_{21}^{\Delta}+I^{0, R}\left(\sigma_{12}+\sigma_{21}\right) \\
& \text { if } k_{-1}^{\Delta} \ll k_{21}^{\Delta}, \omega^{R}=2 k_{-1}^{\Delta}\end{aligned}$ & $\begin{array}{l}\text { Illumination parameters } \\
\qquad \begin{array}{l}\left(\frac{I_{1}^{0}}{I_{2}^{0}}\right)^{R} \simeq \sqrt{\frac{\left(\sigma_{12,2}+\sigma_{21,2}\right) \sigma_{21,2}}{\left(\sigma_{12,1}+\sigma_{21,1}\right) \sigma_{21,1}}} \\
\text { if } k_{-1}^{\Delta} \gg \sigma_{21,2} I_{2}^{0}, \omega^{R} \simeq\left(\left(\sigma_{12,1}+\sigma_{21,1}\right)\left(\frac{I_{1}^{0}}{I_{2}^{0}}\right)^{R}+\left(\sigma_{12,2}+\sigma_{21,2}\right)\right) I_{2}^{0} \\
\text { if } k_{-1}^{\Delta} \ll \sigma_{21,2} I_{2}^{0}, \omega^{R}=2 k_{-1}^{\Delta}\end{array}\end{array}$ \\
\hline
\end{tabular}

Table 5: Summary of the analytical expressions of the control parameters associated with the dynamic titration protocol in one- and two- color systems.

It is worth noting that the protocol is also well adapted to discriminating targeted species with both different values of $k_{+1}^{\Delta}$ and $k_{-1}^{\Delta}$, using the resonance conditions on $F_{\text {tot }}^{R}$ and $\omega^{R}$. When complexation is slower than photoisomerization, the resonance condition setting $\omega^{R}$ involves $k_{-1}^{\Delta}$ and the condition setting $F_{t o t}^{R}$ involves $K_{d}$, i.e. $k_{+1}^{\Delta}$ when $k_{-1}^{\Delta}$ has already been singularized by the choice of $\omega^{R}$. Then the titrating protocol involves the values of both the forward and backward rate constants and not only the equilibrium constant $K_{d}$ : the protocol fully deserves the title of dynamic titration protocol. However, when complexation is faster than photoisomerization, the resonance condition imposing $\omega^{R}$ does not involve $k_{-1}^{\Delta}$, as shown in Eqs. $(65,69)$. In this case, the protocol belongs to the class of standard titrations relying on the variation of the equilibrium constant of reaction between titrated and titrating species.

The conditions on the control parameters to be imposed to implement the dynamic titration protocol are summarized in Table 5 for one- and two- color systems. 

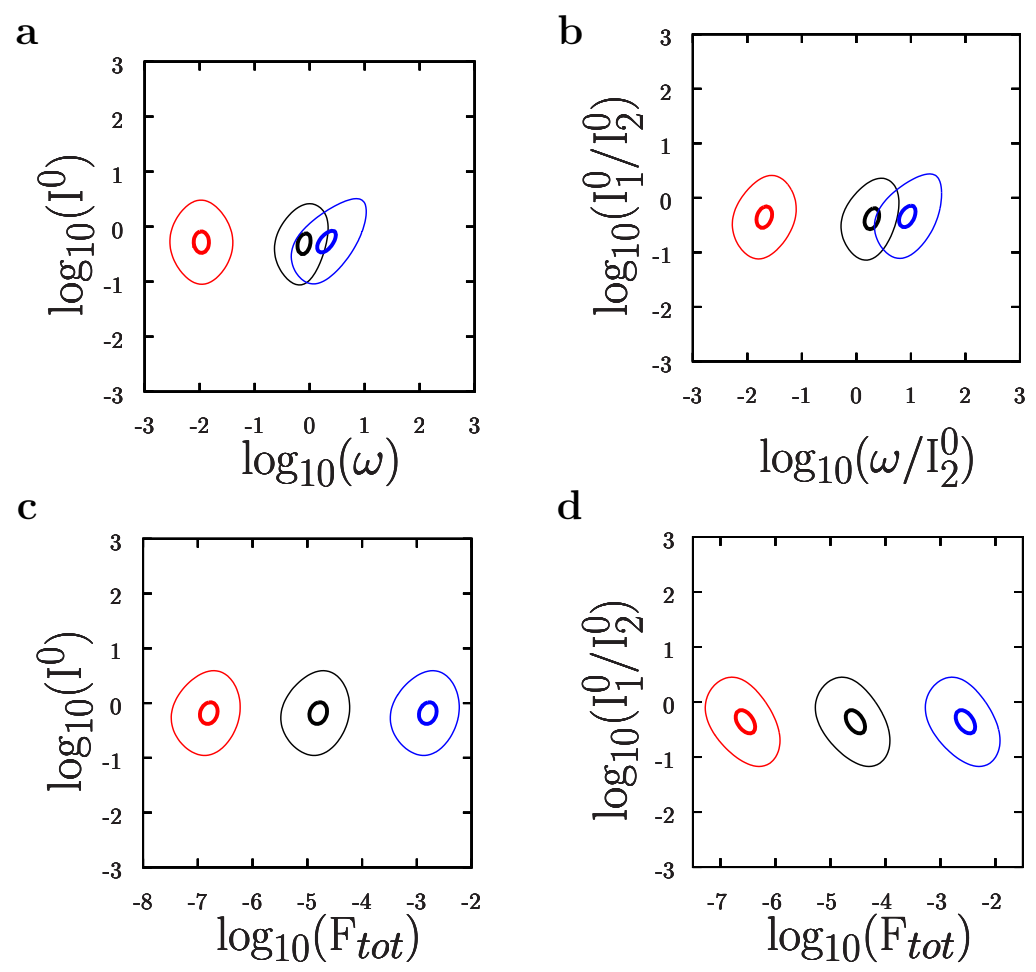

Figure 6: Isodensity curves corresponding to 50\% (thin line) and 95\% (thick line) of the resonant out-of-phase amplitude $1 B^{1, \cos } / N \min \left(R_{t o t}, F_{t o t}\right)$, optimized with respect to the three control parameters, for $R_{t o t}=0.1 K_{d}$. (a,c) One-color system: $\sigma_{12}=\sigma_{21}=1$ in arbitrary units, $k_{21}^{\Delta}=1 \mathrm{~s}^{-1}$. (a) $k_{-1}^{\Delta}=10^{-2} \mathrm{~s}^{-1}$ (red), $k_{-1}^{\Delta}=1 \mathrm{~s}^{-1}$ (black), and $k_{-1}^{\Delta}=10^{2} \mathrm{~s}^{-1}$ (blue), for $K_{d}=100 \mathrm{nM}$, in the $\left(\omega, I^{0}\right)$ space, (c) $K_{d}=1$ $\mathrm{nM}$ (red), $K_{d}=10^{2} \mathrm{nM}$ (black), and $K_{d}=10^{4} \mathrm{nM}$ (blue), for $k_{-1}^{\Delta}=10^{-1} \mathrm{~s}^{-1}$, in the $\left(F_{\text {tot }}, I^{0}\right)$ space, (b,d) Two-color system: $\sigma_{12,1}=\sigma_{21,2}=1$ in arbitrary units, $\sigma_{12,2}=\sigma_{21,1}=5$ in arbitrary units. (b) $k_{-1}^{\Delta} / I_{2}^{0}=10^{-2} \mathrm{~s}^{-1}$ (red), $k_{-1}^{\Delta} / I_{2}^{0}=1 \mathrm{~s}^{-1}$ (black), and $k_{-1}^{\Delta} / I_{2}^{0}=10^{2} \mathrm{~s}^{-1}$ (blue), for $K_{d}=100 \mathrm{nM}$, in the $\left(\omega / I_{2}^{0}, I_{1}^{0} / I_{2}^{0}\right)$ space, (d) $K_{d}=1 \mathrm{nM}$ (red), $K_{d}=10^{2} \mathrm{nM}$ (black), and $K_{d}=10^{4} \mathrm{nM}$ (blue), $k_{-1}^{\Delta} / I_{2}^{0}=10^{-1}$ $\mathrm{s}^{-1}$, in the $\left(F_{\text {tot }}, I_{1}^{0} / I_{2}^{0}\right)$ space.

\section{Conclusion}

A dynamic titration method is proposed for compounds that can react with a reversibly photoswitchable reagent. The principle relies on applying light modulation which imposes forced concentration oscillations. We have chosen to detect the outof-phase amplitude of concentration oscillations. This observable presents satisfying properties for dynamic titration because it is proportional to the total concentration of a species of interest and takes appreciable values only for the targeted species. Sin- 
gularizing a targeted species amounts to setting the control parameters to resonant values that optimize the observable. The resonance conditions consist in adjusting the period of the forced illumination to the relaxation time of the slowest reaction. Moreover they impose that both reactions, photoisomerization and complexation, are at equilibrium at leading order: At the resonance, the chemical system obeys detailed balance. We have derived user-friendly analytical expressions that relate the resonant control parameters to the kinetics of the titrated/titrating couple. The validity of the analytical predictions has been numerically checked. Using these results, we have proposed an experimental protocol able to dynamically titrate any targeted species, provided that the photoswitchable reagent has been adequately chosen.

From an experimental point of view, the implementation of the titration protocol requests to preliminarily study the kinetics of photoswitching and complexation which can be accomplished with standard photochemical and stopped-flow methods. Then, collecting data should not be a problem and data processing is expected to be easy.

The present titration protocol relying on periodic light excitation should yield a better signal-to-noise ratio than titration protocols relying on thermodynamic equilibrium or on the analysis of the response to a jump like in a standard dynamic titration. ${ }^{12}$ Indeed although the observable reaches half the value of the steady concentration in the most favorable case, lock-in detection acts as a band-filter around the resonant angular frequency, which efficiently filters the noise spectrum. As such, this titration protocol should find useful applications in analysis and imaging under demanding conditions.

\section{Conflicts of interest}

There are no conflicts to declare. 


\section{References}

[1] B. N. G. Giepmans, S. R. Adams, M. H. Ellisman, and R. Y. Tsien, Science, 2006, 312, 217-224.

[2] A. Valm, S. Cohen, W. Legant, J. Melunis, U. Hershberg, E. Wait, A. Cohen, M. Davidson, E. Betzig, and J. Lippincott-Schwartz, Nature, 2017, 546, 162-167.

[3] R. Weissleder and M. Nahrendorf, Proc. Natl. Acad. Sci. USA, 2015, 112, 1442414428.

[4] H. Grecco, S. Imtiaz, and E. Zamir, Cytometry Part A, 2016, 89, 761-775.

[5] J. Livet, T. A. Weissman, H. Kang, R. W. Draft, J. Lu, R. A. Bennis, J. R. Sanes, and J. W. Lichtman, Nature, 2007, 450, 56-61.

[6] A. Valm, R. Oldenbourg, and G. Borisy, PLOS One, 2016, 11, e0158495.

[7] S. Pontes-Quero, L. Heredia, V. Casquero-Garcia, M. Fernandez-Chacon, W. Luo, A. Hermoso, M. Bansal, I. Garcia-Gonzalez, M. Sanchez-Munoz, J. Perea, A. Galiana-Simal, I. Rodriguez-Arabaolaza, S. Del Olmo-Cabrera, S. Rocha, L. Criado-Rodriguez, G. Giovinazzo, and R. Benedito, Cell, 2017, 170, 800-814.

[8] M. J. Gerdes, C. J. Sevinsky, A. Sood, S. Adak, M. O. Bello, A. Bordwell, A. Can, A. Corwin, S. Dinn, R. J. Filkins, D. Hollman, V. Kamath, S. Kaanumalle, K. Kenny, M. Larsen, M. Lazare, Q. Li, C. Lowes, C. C. McCulloch, E. McDonough, M. C. Montalto, Z. Pang, J. Rittscher, A. Santamaria-Pang, B. D. Sarachan, M. L. Seel, A. Seppo, K. Shaikh, Y. Sui, J. Zhang, and F. Ginty, Proc. Natl. Acad. Sci. USA, 2013, 110, 11982-11987.

[9] P. Liberali, B. Snijder, and L. Pelkmans, Nat. Rev. Genet., 2015, 16, 18-32.

[10] R. Winkler-Oswatitsch and M. Eigen, Angew. Chem. Intl. Ed., 1979, 18, 20-49. 
[11] J. Quérard, T. Le Saux, A. Gautier, D. Alcor, V. Croquette, A. Lemarchand, C. Gosse, and L. Jullien, ChemPhysChem, 2016, 17, 1396-1413.

[12] M. Eigen and L. de Mayer, Relaxation Methods in Techniques of Organic Chemistry, Wiley, New York, 1963.

[13] C. F. Bernasconi, Relaxation kinetics, Academic Press, New York, 1976.

[14] H. Strehlow and W. Knoche, Fundamentals of chemical relaxation, Weinheim, New York, 1977.

[15] B. Nölting, Protein folding kinetics, Springer, Berlin, 2006.

[16] J. F. Eccleston, S. R. Martin, and M. J. Schilstra, Methods Cell Biol., 2008, $84,445-477$.

[17] V. Petrov, Q. Ouyang, and H. L. Swinney, Nature, 1997, 388, 655-657.

[18] D. Baurecht and U. P. Fringeli, Rev. Sci. Instrum., 2001, 72, 3782-3792.

[19] D. Baurecht, I. Porth, and U. P. Fringeli, Vib. Spectrosc., 2002, 30, 85-92.

[20] V. P. Zhdanov, Surf. Sci. Rep., 2004, 55, 1-48.

[21] J. Dushoff, J. B. Plotkin, S. A. Levin, and D. J. Earn, Proc. Natl. Acad. Sci. U.S.A, 2004, 101, 16915-16916.

[22] M. D. McDonnell and D. Abbott, PLOS Comput. Biol., 2009, 5, e1000348.

[23] H. Berthoumieux, C. Antoine, L. Jullien, and A. Lemarchand, Phys. Rev. E, 2009, 79, 021906.

[24] I. Schoen, H. Krammer, and D. Braun, Proc. Natl. Acad. Sci. U. S. A., 2009, 106, 21649-61654. 
[25] K. Zrelli, T. Barilero, E. Cavatore, H. Berthoumieux, T. Le Saux, V. Croquette, A. Lemarchand, C. Gosse, and L. Jullien, Anal. Chem., 2011, 83, 2476-2484.

[26] M. Platkov and M. Gruebele, J. Chem. Phys., 2014, 141, 035103.

[27] K. Dave, A. Davtyan, G. A. Papoian, M. Gruebele, and M. Platkov, ChemPhysChem, 2016, 17, 1341-1348.

[28] A. Davtyan, M. Platkov, M. Gruebele, and G. A. Papoian, ChemPhysChem, 2016, 17, 1305-1313.

[29] H. Berthoumieux, L. Jullien, and A. Lemarchand, Phys. Rev. E, 2007, 76, 056112.

[30] H. Berthoumieux, C. Antoine, and A. Lemarchand, J. Chem. Phys., 2009, 131, 084106.

[31] A. Lemarchand, H. Berthoumieux, L. Jullien, and C. Gosse, J. Phys. Chem. A, 2012, 116, 8455-8463.

[32] F. Closa, C. Gosse, L. Jullien, and A. Lemarchand, J. Chem. Phys., 2013, 138, 244109.

[33] F. Closa, C. Gosse, L. Jullien, and A. Lemarchand, J. Chem. Phys., 2015, 142, 174108.

[34] A. Gautier, C. Gauron, M. Volovitch, D. Bensimon, L. Jullien, and S. Vriz, Nat. Chem. Biol., 2014, 10, 533-541.

[35] P. Wang, J. Querard, S. Maurin, S. S. Nath, T. Le Saux, A. Gautier, and L. Jullien, Chem. Sci., 2013, 4, 2865-2873.

[36] M. A. Plamont, E. Billon-Denis, S. Maurin, C. Gauron, F. M. Pimenta, C.G. Specht, J. Shi, J. Qurard, B. Pan, J. Rossignol, K. Moncoq, N. Morellet, M. 
Volovitch, E. Lescop, Y. Chen, A. Triller, S. Vriz, T. Le Saux, L. Jullien, and A. Gautier, Proc. Natl. Acad. Sci. U.S.A, 2016, 133, 497-502.

[37] F. M. Pimenta, G. Chiappetta, T. Le Saux, J. Vinh, L. Jullien, and A. Gautier, Sci. Rep., 2017, 7, 12316.

[38] H. Bouas-Laurent and H. Dürr, Pure Appl. Chem., 2001, 73, 639-665.

[39] M. S. Wang, G. Xu, Z. J. Zhang, and G. C. Guo, Chem. Commun., 2010, 46, $361-376$.

[40] T. Fukaminato, J. Photochem. Photobiol. C : Photochem. Rev., 2011, 12, $177-$ 208.

[41] S. van de Linde and M. Sauer, Chem. Soc. Rev., 2014, 43, 1076-1087.

[42] D. Bourgeois and V. Adam, IUBMB Life, 2012, 64, 482-491.

[43] X. X. Zhou and M. Z. Lin, Curr. Opin. Chem. Biol., 2013, 17, 682-690.

[44] H. M. Bandara and S. C. Burdette, Chem. Soc. Rev., 2012, 41, 1809-1825.

[45] M. Dong, A. Babalhavaeji, S. Samanta, A. A. Beharry, and G. A. Woolley, Acc. Chem. Res., 2015, 48, 2662-2670.

[46] D. Bleger and S. Hecht, Angew. Chem., Int. Ed., 2015, 54, 11338-11349.

[47] J. Widengren and P. Schwille, J. Phys. Chem. A, 2000, 104, 6416-2428.

[48] G. T. Dempsey, M. Bates, W. E. Kowtoniuk, D. R. Liu, R. Y. Tsien, and X. Zhuang, J. Am. Chem. Soc., 2009, 131, 18192-18193.

[49] H. Tian and S. Yanga, Chem Soc. Rev., 2004, 33, 85-97.

[50] K. Matsuda and M. Irie, J. Photochem. Photobiol. C, Photochem. Rev., 2004, 5, 169-182. 
[51] C. Yun, J. You, J. Kim, J. Huh, and E. Kim, J. Photochem. Photobiol. C, Photochem. Rev., 2009, 10, 111-129.

[52] R. Klajn, Chem. Soc. Rev., 2014, 43, 148-184.

[53] J. Quérard, T. Z. Markus, M. A. Plamont, C. Gauron, P. Wang, A. Espagne, M. Volovitch, S. Vriz, V. Croquette, A. Gautier, T. Le Saux, and L. Jullien, Angew. Chem. Int. Ed., 2015, 127, 2671-2675.

[54] J. Quérard, R. Zhang, Z. Kelemen, M. A. Plamont, X. Xie, R. Chouket, I. Roemgens, Y. Korepina, S. Albright, E. Ipendey, M. Volovitch, H. L. Sladitschek, P. Neveu, L. Gissot, A. Gautier, J. D. Faure, V. Croquette, T. Le Saux, and L. Jullien, Nat. Commun., 2017, 8, 969. 